Supporting Information

\title{
Porphyrins Bearing Stable meso-Alkylidenyl Double Bonds. A New Family of Non-planar Porphyrinoids
}

Eun-Kyung Sim, ${ }^{\dagger}$ Seung-Doo Jeong, ${ }^{\dagger}$ Dae-Wi Yoon, ${ }^{\dagger}$ Seong-Jin Hong, ${ }^{\dagger}$ Youngjin Kang ${ }^{\ddagger}$ and Chang-Hee

$$
\text { Lee }^{*},
$$

${ }^{*}$, Department of Chemistry, Kangwon National University Chun-Chon 200-701, Korea and

${ }^{\ddagger}$ Department of Science Education, Kangwon National University Chun-Chon 200-701, Korea. 


\section{Table of contents}

Experimental --------- S2-S4

${ }^{1} \mathrm{H}$ NMR, ${ }^{13} \mathrm{C}$ NMR and HRMS spectra of compound (10) ---

${ }^{1} \mathrm{H}$ NMR, ${ }^{13} \mathrm{C}$ NMR and HRMS spectra of compound (11) --------------------- S8-S10

${ }^{1} \mathrm{H}$ NMR, ${ }^{13} \mathrm{C}$ NMR and HRMS spectra of compound (12) --

${ }^{1} \mathrm{H}$ NMR, ${ }^{13} \mathrm{C}$ NMR and HRMS spectra of compound (13) -------------------- S14-S16

Crystal data, structure refinement, atomic coordinates, bond distance, bond angles, and anisotropic

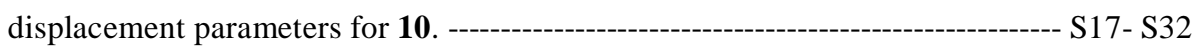

Crystal data, structure refinement, atomic coordinates, bond distance, bond angles, and anisotropic displacement parameters for $\mathbf{1 1}$.

S33-S44 


\section{Experimental Section}

Proton NMR spectra (400 MHz, Bruker DPX-400) were recorded using TMS as the internal standard. High resolution mass spectra were obtained on an Voyager-DE STR MALDI-TOF mass spectrometer. Column chromatography was performed over silica gel (Merck, 230-400 mesh). Pyrrole was distilled at atmospheric pressure from $\mathrm{CaH}_{2}$. All other reagents were obtained from Aldrich and used as received unless noted otherwise. Compounds 4, 5, 6, 7, 8 and 9 were synthesized by slight modifying the reported procedure. ${ }^{12,13}$

\section{6,21-Bis(diethoxy carbonylmethylidene)-11,16-diphenyl-24-oxabenziporphyrin (10)}

Compound 6 ( $0.11 \mathrm{~g}, 0.2 \mathrm{mmole})$ and compound 8 (0.062 g, $0.22 \mathrm{mmole})$ were dissolved in $\mathrm{CH}_{3} \mathrm{CN}(20 \mathrm{~mL})$ with stirring, then TFA $(0.04 \mathrm{~mL}, 0.52 \mathrm{mmole})$ were added. The whole mixture was stirred for $24 \mathrm{~h}$ at $25{ }^{\circ} \mathrm{C}$. Then, DDQ $(0.141 \mathrm{~g}, 0.62 \mathrm{mmole})$ and TEA $(0.10 \mathrm{~mL}$, 0.72 mmole) were added. The mixture was stirred for $1 \mathrm{~h}$ and extracted with $\mathrm{CH}_{2} \mathrm{Cl}_{2}$ after adding brine $(40 \mathrm{~mL})$. The organic layer was dried $\left(\mathrm{Na}_{2} \mathrm{SO}_{4}\right)$ and solvent was removed in vacuo. The mixture was chromatographed on silica, eluting first with $\mathrm{CH}_{2} \mathrm{Cl}_{2} / \mathrm{EtOAc}$ (19/1) to afford the crude mixture of compound (10) and (12). The isolation of (10) and (12) was accomplished by repeated column chromatography on silica (EtOAc/hexanes $=1 / 2)$. Compound $(\mathbf{1 0})$ was eluted first followed by compound (12). For compound (10); Yield: $0.032 \mathrm{~g} \mathrm{(20} \mathrm{\% );}{ }^{1} \mathrm{H}$ NMR $\left(400 \mathrm{MHz}, \mathrm{CDCl}_{3}\right) \delta 8.02$ (br s, 2H, NH), 7.49-7.20 (m, 4H, Ar-H, 10H, Ar-H), 6.71-6.68 (m, $2 \mathrm{H}$, pyrrole-H), $6.18(\mathrm{~s}, 2 \mathrm{H}$, furan-H), 5.77-5.75 (m, 2H, pyrrole-H), 4.29 (q, $J=7.13 \mathrm{~Hz}, 4 \mathrm{H}$, $\left.\mathrm{CH}_{2}\right), 4.06$ (q, $\left.J=7.11 \mathrm{~Hz}, 4 \mathrm{H}, \mathrm{CH}_{2}\right), 1.29$ (t, $\left.J=7.13 \mathrm{~Hz}, 6 \mathrm{H}, \mathrm{CH}_{3}\right), 1.13$ (t, $J=7.11 \mathrm{~Hz}, 6 \mathrm{H}$, $\left.\mathrm{CH}_{3}\right) ;{ }^{13} \mathrm{C}$ NMR $\left(100 \mathrm{MHz}, \mathrm{CDCl}_{3}\right) \delta 167.58,164.06,154.63,143.42,138.41,135.16,132.16$, $131.47,128.74,128.64,128.25,127.94,126.50,124.88,120.25,115.52,115.21,108.10,51.58$, 50.88, 13.92, 13.89; MALDI-TOF MS Calcd. for $\mathrm{C}_{48} \mathrm{H}_{42} \mathrm{~N}_{2} \mathrm{O}_{9}$ 790.2890, Found 791.3417.

\section{6,21-Bis(diethoxycarbonylmethylidene)-11,16-diphenyl-24-oxapyriporphyrin (11)}

Compound 7 (0.214 g, 0.39 mmole) and compound 8 (0.109 g, 0.39 mmole) were dissolved in $\mathrm{CH}_{3} \mathrm{CN}(39 \mathrm{~mL})$ with stirring and then $\mathrm{NH}_{4} \mathrm{Cl}(0.208 \mathrm{~g}, 3.89$ mmole $)$ and TFA $(0.105 \mathrm{~mL}, 1.36$ mmoel) were added. The whole mixture was stirred for $2 \mathrm{~h}$ at $25{ }^{\circ} \mathrm{C}$ and DDQ (0.266 g, 1.17 mmole) and TEA ( $0.23 \mathrm{~mL}, 1.65 \mathrm{mmole})$ was added successively. The mixture was stirred for 1 $\mathrm{h}$ and then combined with brine $(40 \mathrm{~mL})$. The mixture was extracted with $\mathrm{CH}_{2} \mathrm{Cl}_{2}$ and the organic layer was dried $\left(\mathrm{Na}_{2} \mathrm{SO}_{4}\right)$. Solvent was removed in vacuo and the remaining solid was separated by column chromatography on silica (EtOAc/hexanes=1/2). Yield: $0.063 \mathrm{~g}(20 \%)$; UV-Vis. $\left(\mathrm{CH}_{2} \mathrm{Cl}_{2}\right) \lambda_{\max }(\log \varepsilon) 392$ (4.48), 539 (4.23); ${ }^{1} \mathrm{H}$ NMR (300 MHz, $\left.\mathrm{CDCl}_{3}\right) \delta 8.09$ (br s, 2H, NH), 7.76 (t, $J=7.76 \mathrm{~Hz}, 1 \mathrm{H}$, pyridine-H), 7.40 (d, $J=7.76 \mathrm{~Hz}, 2 \mathrm{H}$, pyridine-H), 7.38-7.26 (m, 10H, benzene-H), 6.74-6.17 (m, 2H, pyrrole-H), 6.17 (s, 2H, furan-H), 5.80-5.78 (m, 2H, 
pyrrole-H), 4.29 (q, $\left.J=7.13 \mathrm{~Hz}, 4 \mathrm{H}, \mathrm{CH}_{2}\right), 4.07$ (q, $\left.J=7.10 \mathrm{~Hz}, 4 \mathrm{H}, \mathrm{CH}_{2}\right), 1.28(\mathrm{t}, J=7.13 \mathrm{~Hz}$, $\left.6 \mathrm{H}, \mathrm{CH}_{3}\right), 1.15\left(\mathrm{t}, J=7.10 \mathrm{~Hz}, 6 \mathrm{H}, \mathrm{CH}_{3}\right) ;{ }^{13} \mathrm{C} \mathrm{NMR}\left(100 \mathrm{MHz}, \mathrm{CDCl}_{3}\right) \delta 167.58,163.42$, 156.09, 154.41, 142.01, 138.24, 136.46, 135.46, 131.46, 128.28, 127.93, 126.29, 123.57, 121.15, 115.70, 115.57, 108.05, 61.66, 60.99, 13.97, 13.90; MALDI-TOF MS Calcd. for $\mathrm{C}_{47} \mathrm{H}_{41} \mathrm{~N}_{3} \mathrm{O}_{9}$ 791.2843, Found 791.3350.

6,21-Bis(diethoxycarbonylmethylidene)-11,16-di(p-tolyl)-24-thiapyriporphyrin (12), 6,21bis(diethylmalonyl)-11,16-di-( $p$-tolyl)-24-thiapyriporphyrin (13),

Compound 7 (0.324 g, 0.59 mmole), compound 3 (0.228 g, 0.7 mmole), $\mathrm{NH}_{4} \mathrm{Cl}(0.313 \mathrm{~g}, 0.59$ mmol), TFA (0.16 mL, 2.05 mmole), DDQ (0.465 g, 2.05 mmole), and TEA (0.24 mL, 2.34 mmole) were treated identically as for the synthesis of $\mathbf{1 1}$. The mixture was chromatographed on silica, eluting first with $\mathrm{CH}_{2} \mathrm{Cl}_{2} / \mathrm{EtOAc}(9 / 1)$ and then with EtOAc/hexanes (1/2) in order to isolate two different products $\mathbf{1 2}$ and $\mathbf{1 3 .}$

For compound 12; Yield: $0.047 \mathrm{~g}(10 \%)$; UV-Vis. $\left(\mathrm{CH}_{3} \mathrm{CN}\right) \lambda_{\max }(\log \varepsilon) 382$ (4.60), 555 (4.26); ${ }^{1} \mathrm{H}$ NMR $\left(300 \mathrm{MHz}, \mathrm{CDCl}_{3}\right) \delta 8.63$ (br s, $\left.2 \mathrm{H}, \mathrm{NH}\right), 7.86$ (t, $J=7.76 \mathrm{~Hz}, 1 \mathrm{H}$, pyridine-H), 7.42 (d, $J=7.76 \mathrm{~Hz}, 2 \mathrm{H}$, pyridine-H), 7.24-7.16 (m, 8H, benzene-H), 6.75-6.72 (m, $2 \mathrm{H}$, pyrrole-H), $6.61(\mathrm{~s}, 2 \mathrm{H}$, thiophene- $\mathrm{H}), 6.07-6.05(\mathrm{~m}, 2 \mathrm{H}$, pyrrole- $\mathrm{H}), 4.41-4.28(\mathrm{~m}, 4 \mathrm{H}$, $\mathrm{CH}_{2}$ ), 4.09 (q, $\left.J=7.10 \mathrm{~Hz}, 4 \mathrm{H}, \mathrm{CH}_{2}\right), 2.39\left(\mathrm{~s}, 6 \mathrm{H}\right.$, tolyl- $\left.\mathrm{CH}_{3}\right), 1.31$ (t, $J=7.13 \mathrm{~Hz}, 6 \mathrm{H}$, $\mathrm{CH}_{2} \mathrm{CH}_{3}$ ), 1.16 (t, $\left.J=7.10 \mathrm{~Hz}, 6 \mathrm{H}, \mathrm{CH}_{2} \mathrm{CH}_{3}\right) ;{ }^{13} \mathrm{C}$ NMR $\left(100 \mathrm{MHz}, \mathrm{CDCl}_{3}\right) \delta 167.44,162.83$, $155.84,140.59$, 138.20, 136.73, 136.64, 136.49, 135.19, 132.69, 130.61, 130.46, 129.00, 124.04, 122.53, 121.42, 116.71, 114.79, 61.80, 60.97, 21.29, 13.97, 13.90; MALDI-TOF MS Calcd. for $\mathrm{C}_{49} \mathrm{H}_{45} \mathrm{~N}_{3} \mathrm{O}_{9} \mathrm{~S}$ 835.29, Found 834.83.

For compound 13; Yield: $0.008 \mathrm{~g}(2 \%)$; UV-Vis. $\left(\mathrm{CH}_{3} \mathrm{CN}\right) \lambda_{\max }(\log \varepsilon) 395$ (4.59) 578 (4.14); ${ }^{1} \mathrm{H}$ NMR (300 MHz, $\left.\mathrm{CDCl}_{3}, 25 \square\right) \delta 7.73$ (t, $J=7.76 \mathrm{~Hz}, 1 \mathrm{H}$, pyridine-H), 7.30-7.21 (m, 8H, benzene-H), 7.19 (d, $J=7.76 \mathrm{~Hz}, 2 \mathrm{H}$, pyridine-H), 6.81-6.77 (m, 4H, pyrrole-H), 6.79 (s, $2 \mathrm{H}$, thiophene-H), 4.39-4.22 (m, 4H, $\left.\mathrm{CH}_{2}\right), 4.13$ (q, $\left.J=6.82 \mathrm{~Hz}, 4 \mathrm{H}, \mathrm{CH}_{2}\right), 2.42$ (s, 6H, tolyl$\left.\mathrm{CH}_{3}\right), 1.29$ (t, $\left.J=7.14 \mathrm{~Hz}, 6 \mathrm{H}, \mathrm{CH}_{2} \underline{\mathrm{CH}}_{3}\right), 1.12$ (t, $\left.J=7.12 \mathrm{~Hz}, 6 \mathrm{H}, \mathrm{CH}_{2} \underline{\mathrm{CH}}_{3}\right) ;{ }^{1} \mathrm{H}$ NMR (400 $\left.\mathrm{MHz}, \mathrm{CDCl}_{3}, 0{ }^{\circ} \mathrm{C}\right) \delta 7.80(\mathrm{t}, J=7.75 \mathrm{~Hz}, 1 \mathrm{H}$, pyridine- $\mathrm{H}), 7.33-7.28(\mathrm{~m}, 8 \mathrm{H}$, benzene-H), 7.25 (d, $J=7.75 \mathrm{~Hz}, 2 \mathrm{H}$, pyridine-H), 6.85-6.82 (m, 4H, pyrrole-H), 6.84 (s, 2H, thiophene-H), 5.18 (s, 2H, CHCO), 4.40-4.35 (m, 2H, $\mathrm{CH}_{2}$ ), 4.29-4.24 (m, 2H, $\mathrm{CH}_{2}$ ), 4.16-4.08 (m, 4H, $\mathrm{CH}_{2}$ ), 2.44 (s, 6H, tolyl- $\mathrm{CH}_{3}$ ), 1.39 (t, $\left.J=7.12 \mathrm{~Hz}, 6 \mathrm{H}, \mathrm{CH}_{2} \underline{\mathrm{CH}}_{3}\right), 1.15$ (t, $\left.J=7.11 \mathrm{~Hz}, 6 \mathrm{H}, \mathrm{CH}_{2} \underline{\mathrm{CH}}_{3}\right) ;{ }^{13} \mathrm{C}$ NMR $\left(100 \mathrm{MHz}, \mathrm{CDCl}_{3}\right) \delta 167.45,166.34,163.61,158.35,154.99,153.32,146.99,146.62$, 139.56, 135.20, 135.02, 134.07, 130.92, 129.69, 128.61, 128.01, 123.35, 61.95, 61.52, 21.38, 13.91, 13.77; MALDI-TOF MS Calcd. for $\mathrm{C}_{49} \mathrm{H}_{45} \mathrm{~N}_{3} \mathrm{O}_{9} \mathrm{~S}$ exact mass 835.29, Found 834.91. 
${ }^{1} \mathrm{H}$ NMR spectrum of 6,21-bis(diethoxycarbonylmethylidene)-11,16-diphenyl-24-oxabenziporphyrin 10 in $\mathrm{CDCl}_{3}$
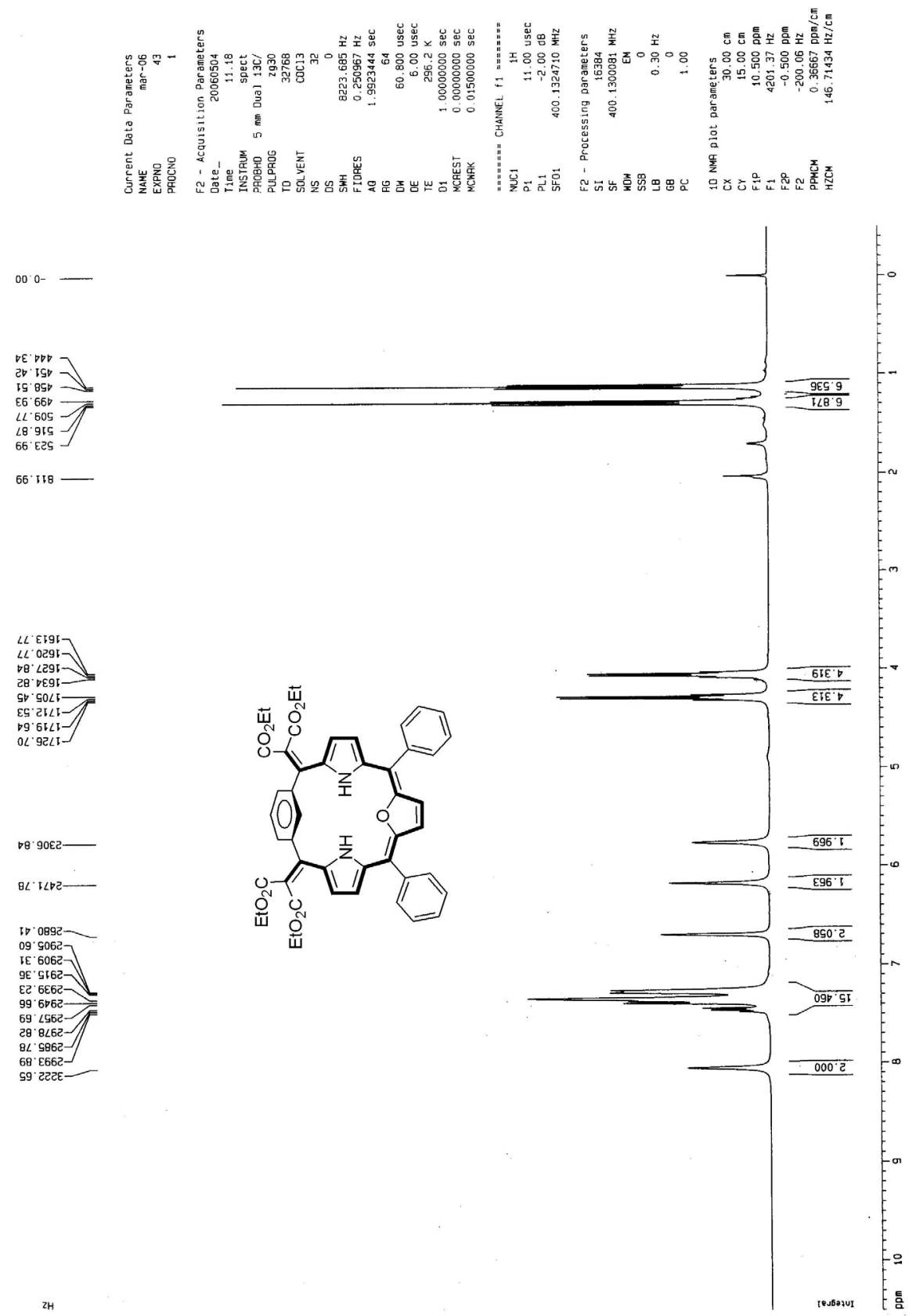
${ }^{13} \mathrm{C} \quad$ NMR spectrum of 6,21-bis(diethoxycarbonylmethylidiene)-11,16-diphenyl-24oxabenziporphyrin 10 in $\mathrm{CDCl}_{3}$

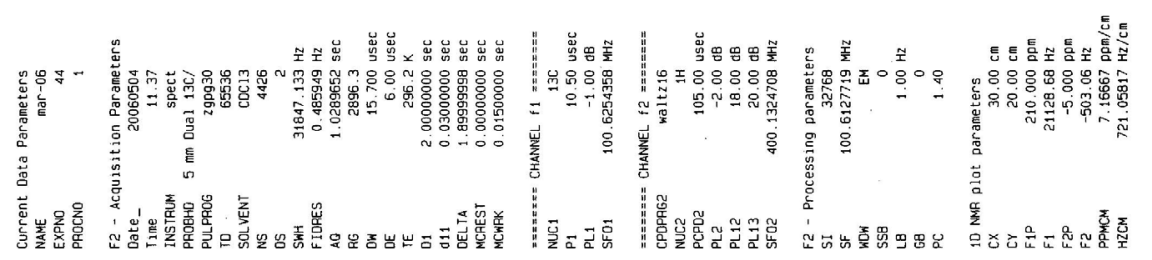

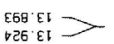

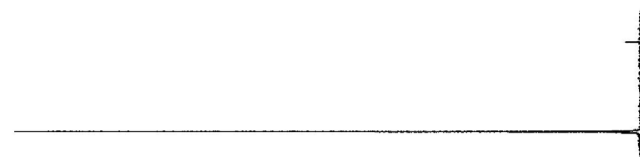

$6 / 8 \cdot 09=$

$\angle D L \cdot 9 L>$

29E: $\angle L=$

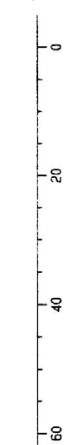

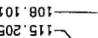

969.05

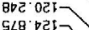

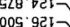

$006.211-$

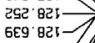

$0 \circ L$
$895 \mathrm{~L}$

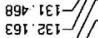

SLI TEL-

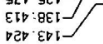

$829 \cdot 091$

$290 \cdot 091-$

089 $291-$
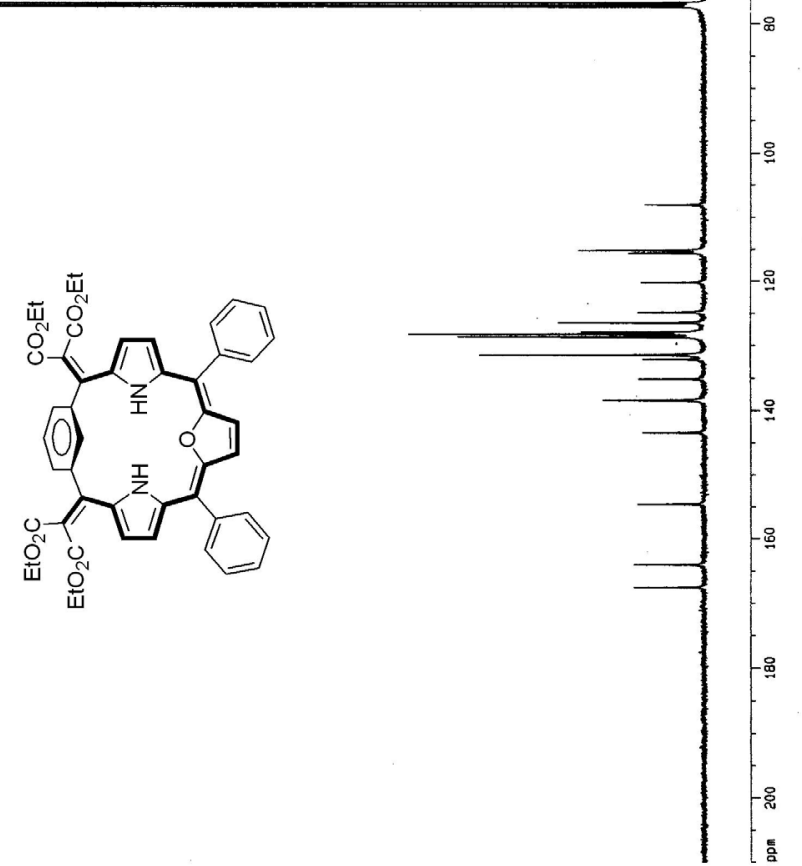
MALDI-TOP spectrum of 6,21-bis(diethoxycarbonylmethylidiene)-11,16-diphenyl-24oxabenziporphyrin 10 in $\mathrm{CDCl}_{3}$
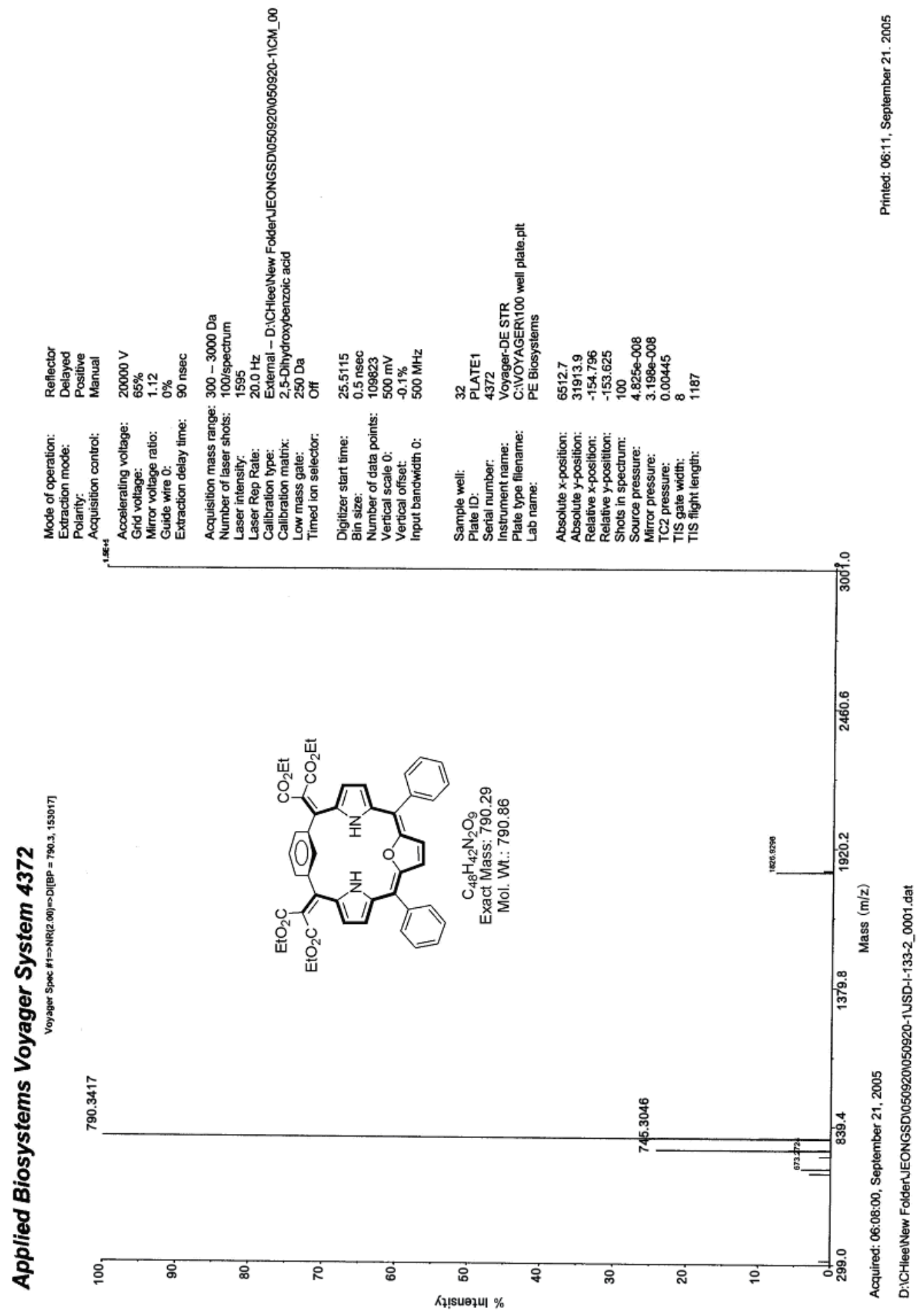
${ }^{1} \mathrm{H}$ NMR spectrum of 6,21-bis(diethoxycarbonylmethylidene)-11,16-diphenyl-24-oxapyriporphyrin 11 in $\mathrm{CDCl}_{3}$
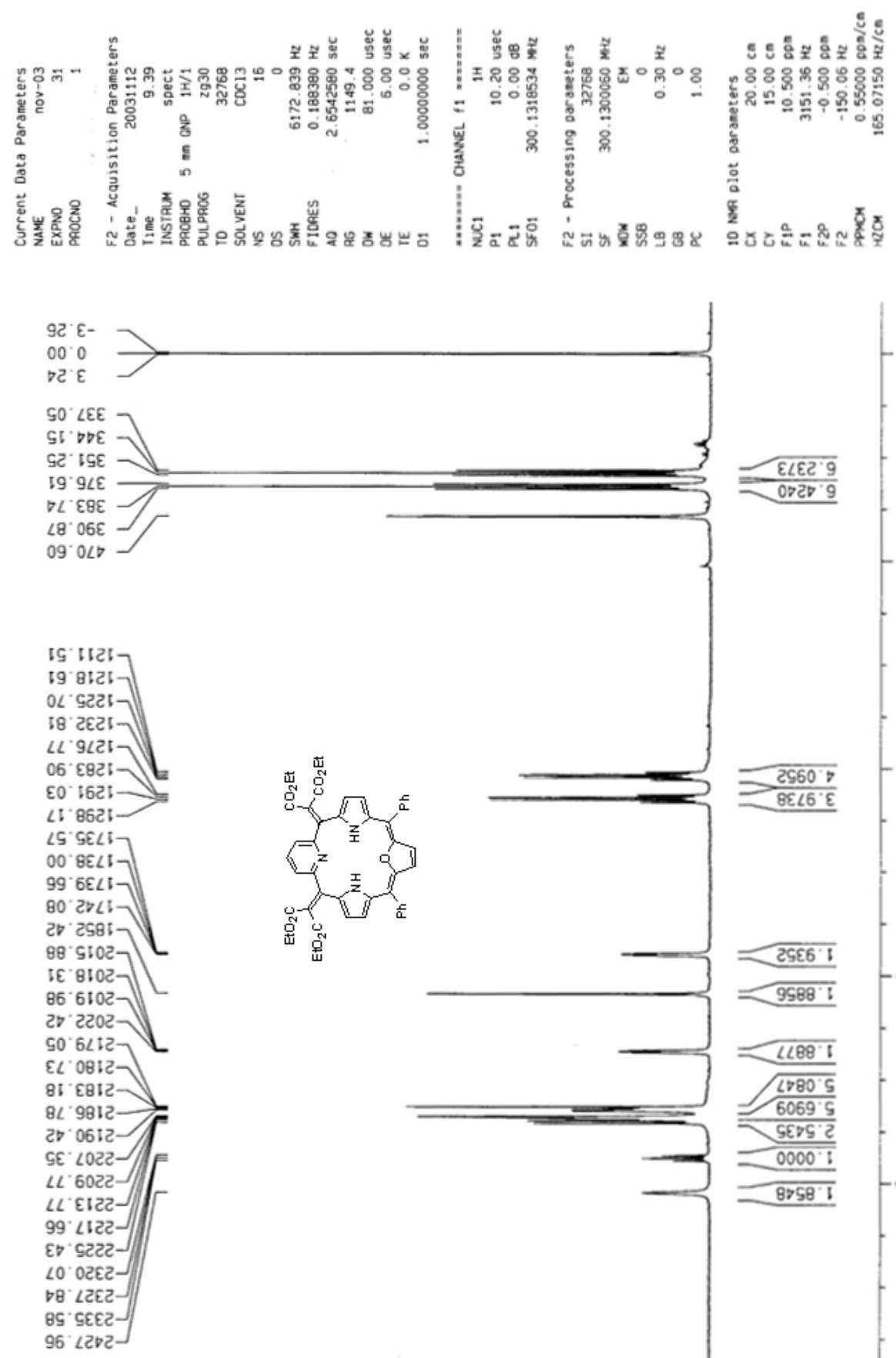

$2 \mathrm{H}$

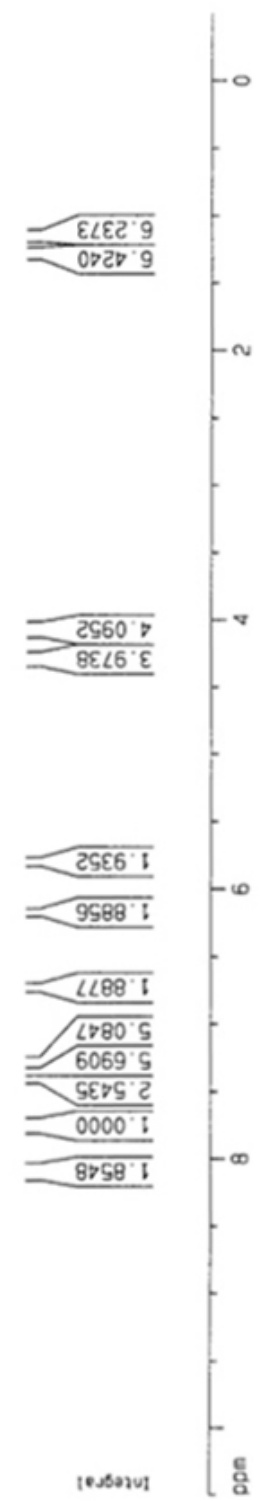


${ }^{13} \mathrm{C}$ NMR spectrum of 6,21-bis(diethoxycarbonylmethylidene)-11,16-diphenyl-24-oxapyriporphyrin $\mathbf{1 1}$ in $\mathrm{CDCl}_{3}$
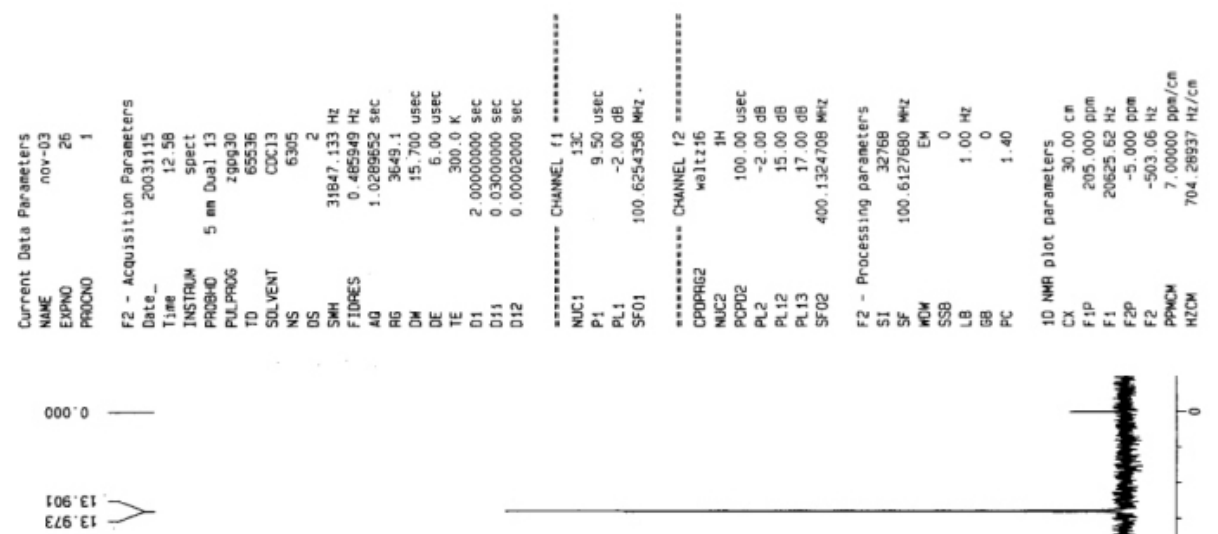

${ }_{096}^{069} 19>$

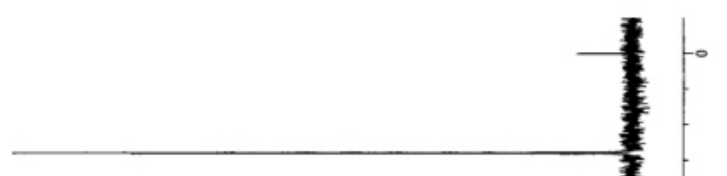

$26 \mathrm{kl}$

900
620

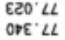

800. $80 \mathrm{t}-$

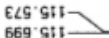

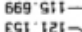

Est $121-$

1652.9517

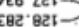

о9p ' $\mathrm{tel}$

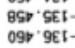

962 eE:-

oto 2015

060. $00^{\circ} 9 \mathrm{SI}^{\circ}=$

เ20 69 -

695 ' 291

-
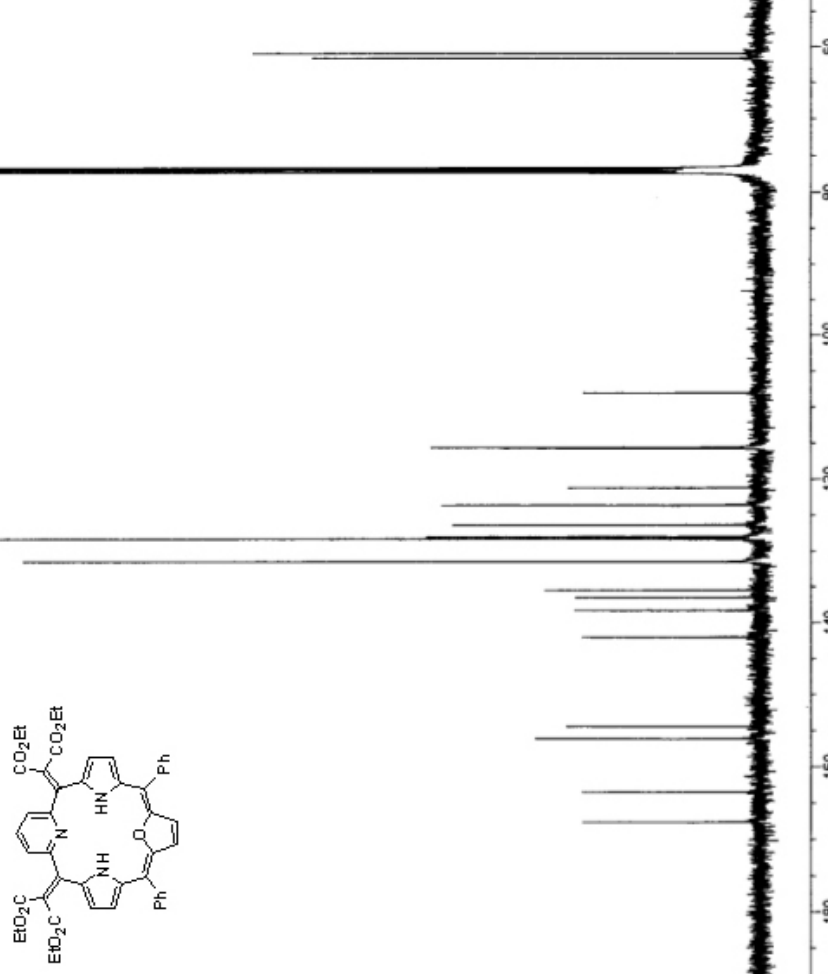
MALDI-TOF spectrum of 6,21-bis(diethoxycarbonylmethylidene )-11,16-diphenyl-24-oxapyriporphyrin 11 in $\mathrm{CH}_{2} \mathrm{Cl}_{2}$

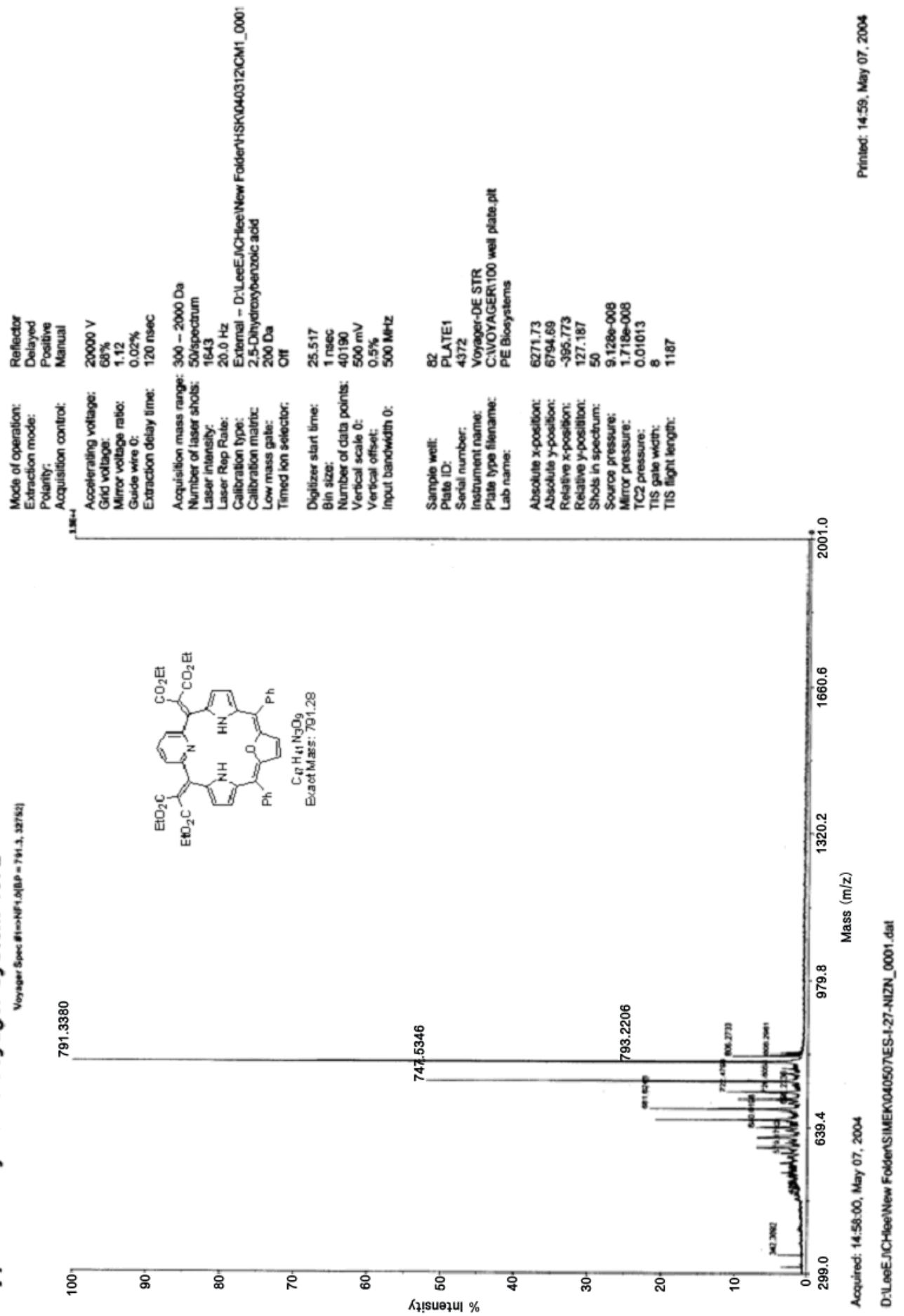


${ }^{1} \mathrm{H}$ NMR spectrum of 6,21-bis(diethoxycarbonylmethylidene)-11,16-di(p-tolyl)-24-thiapyriporphyrin 12 in $\mathrm{CDCl}_{3}$
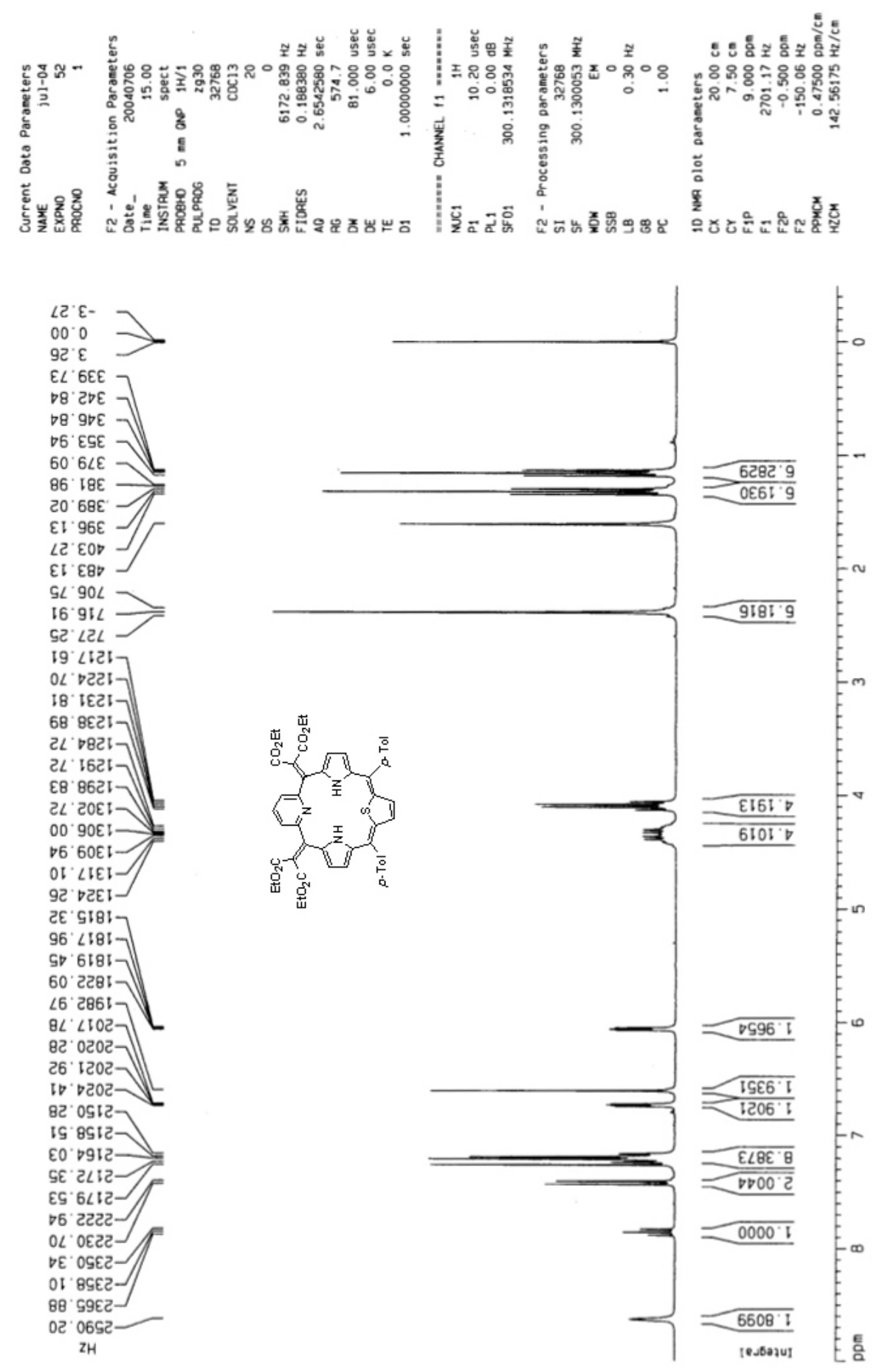
${ }^{13} \mathrm{C}$ NMR spectrum of 6,21-bis(diethoxycarbonylmethylidene)-11,16-di(p-tolyl)-24-thiapyriporphyrin 12 in $\mathrm{CDCl}_{3}$
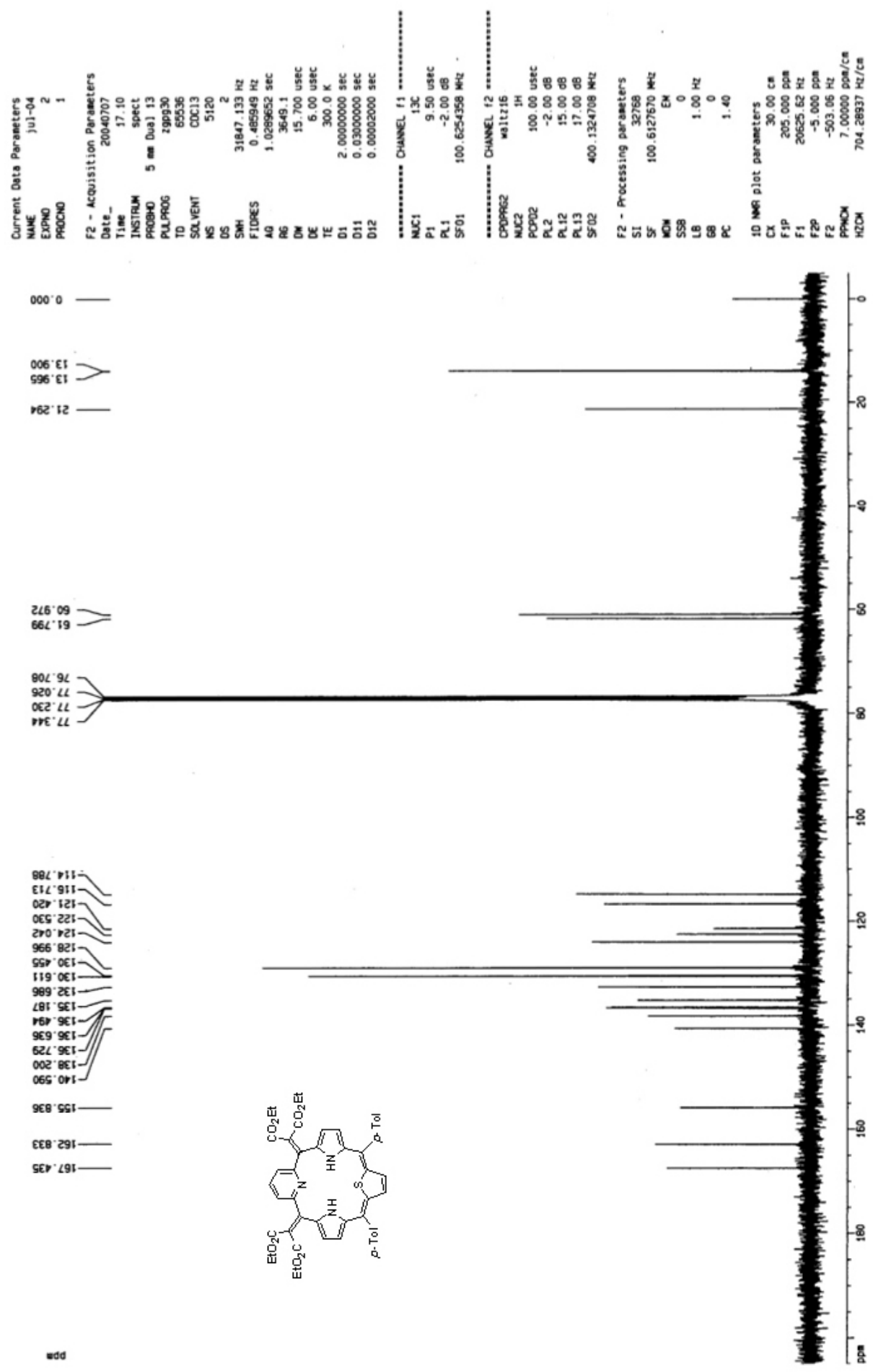
MALDI-TOF spectrum of 6,21-bis(diethoxycarbonylmethylidene)-11,16-di(p-tolyl)-24-thiapyriporphyrin 12 in $\mathrm{CH}_{2} \mathrm{Cl}_{2}$
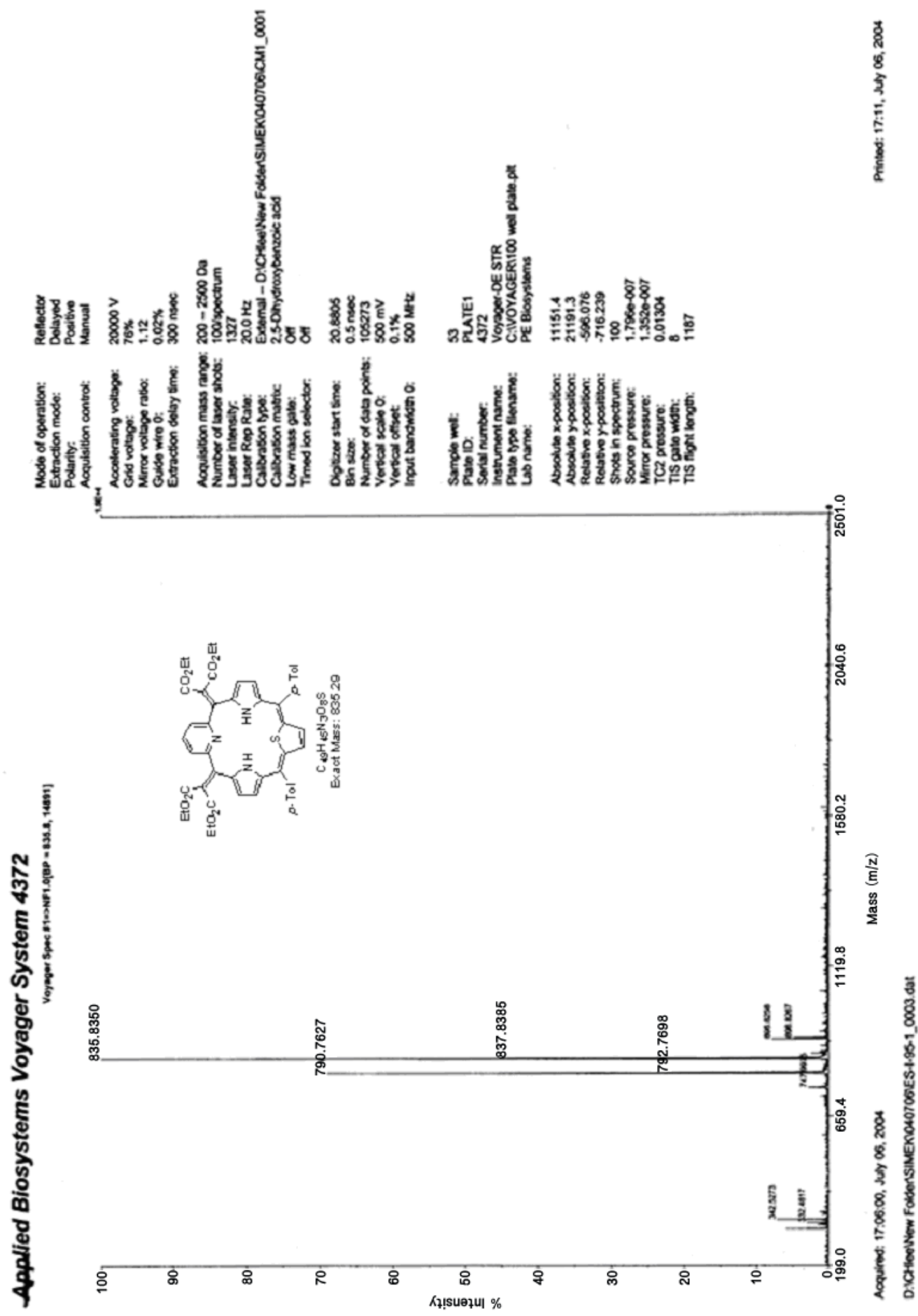
${ }^{1} \mathrm{H}$ NMR spectrum of 6,21-bis(diethylmalonyl)-11,16-di(p-tolyl)-24-thiapyriporphyrin 13 in $\mathrm{CDCl}_{3}$

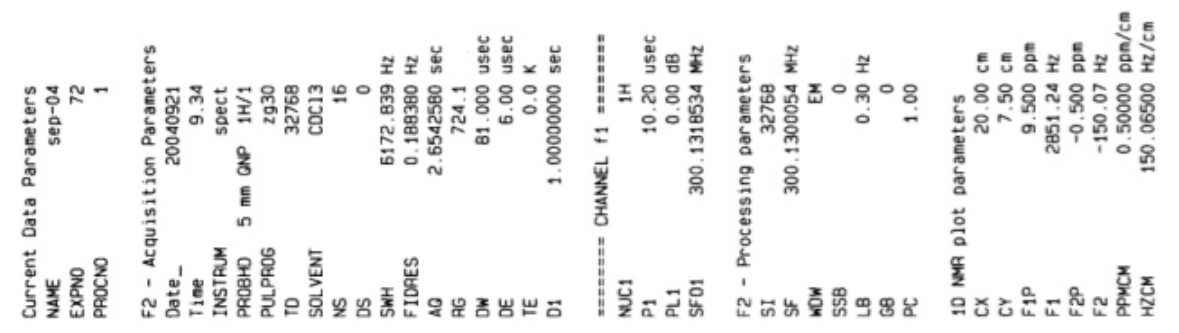

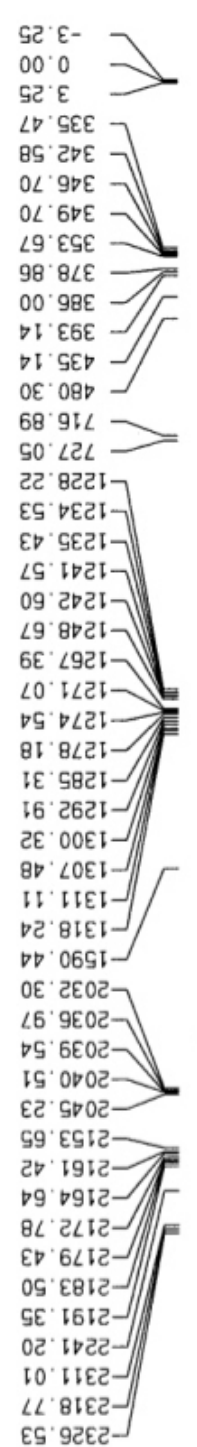

ZH

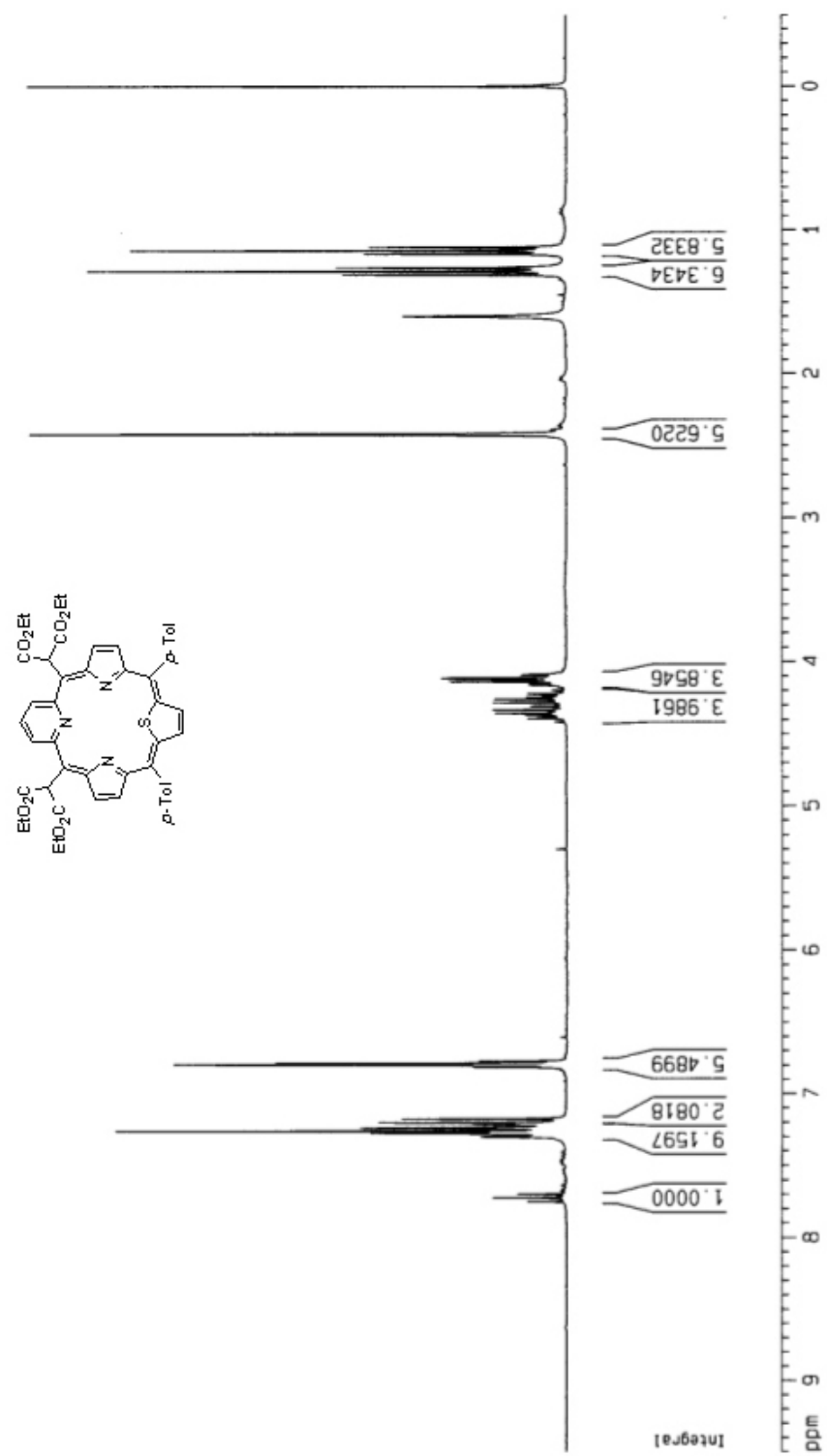


${ }^{13} \mathrm{C}$ NMR spectrum of 6,21-bis(diethylmalonyl)-11,16-di(p-tolyl)-24-thiapyriporphyrin 13 in $\mathrm{CDCl}_{3}$
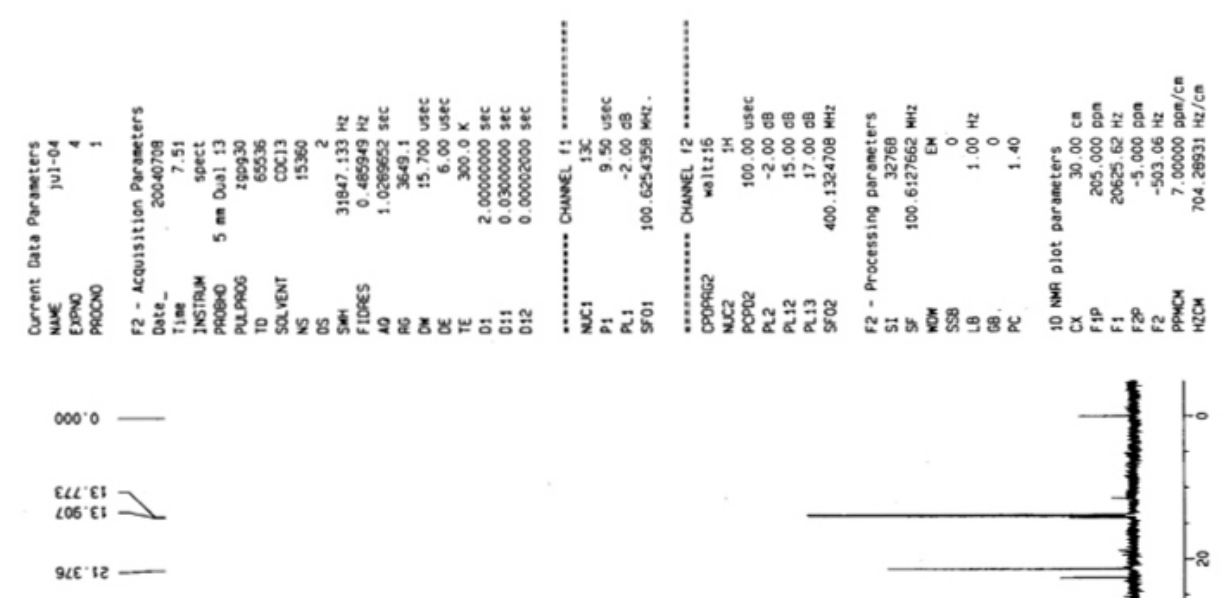

sts.19 7

676 : 9

$\operatorname{lic} \cdot 9 L$

9є2. $U$ -

are' $u$ -

Ise. 1517

119.821

$569.621-$

025.061

$\checkmark \angle 0^{\circ} \mathrm{VE}$

$220: 561-$
L65: 581

BSS GE: -

r29.9p:-

$666.9 \mathrm{ri}>$

$P E$ ' ES: -

6re' $85:-$

909 ' $191-$

IrE. $991-$
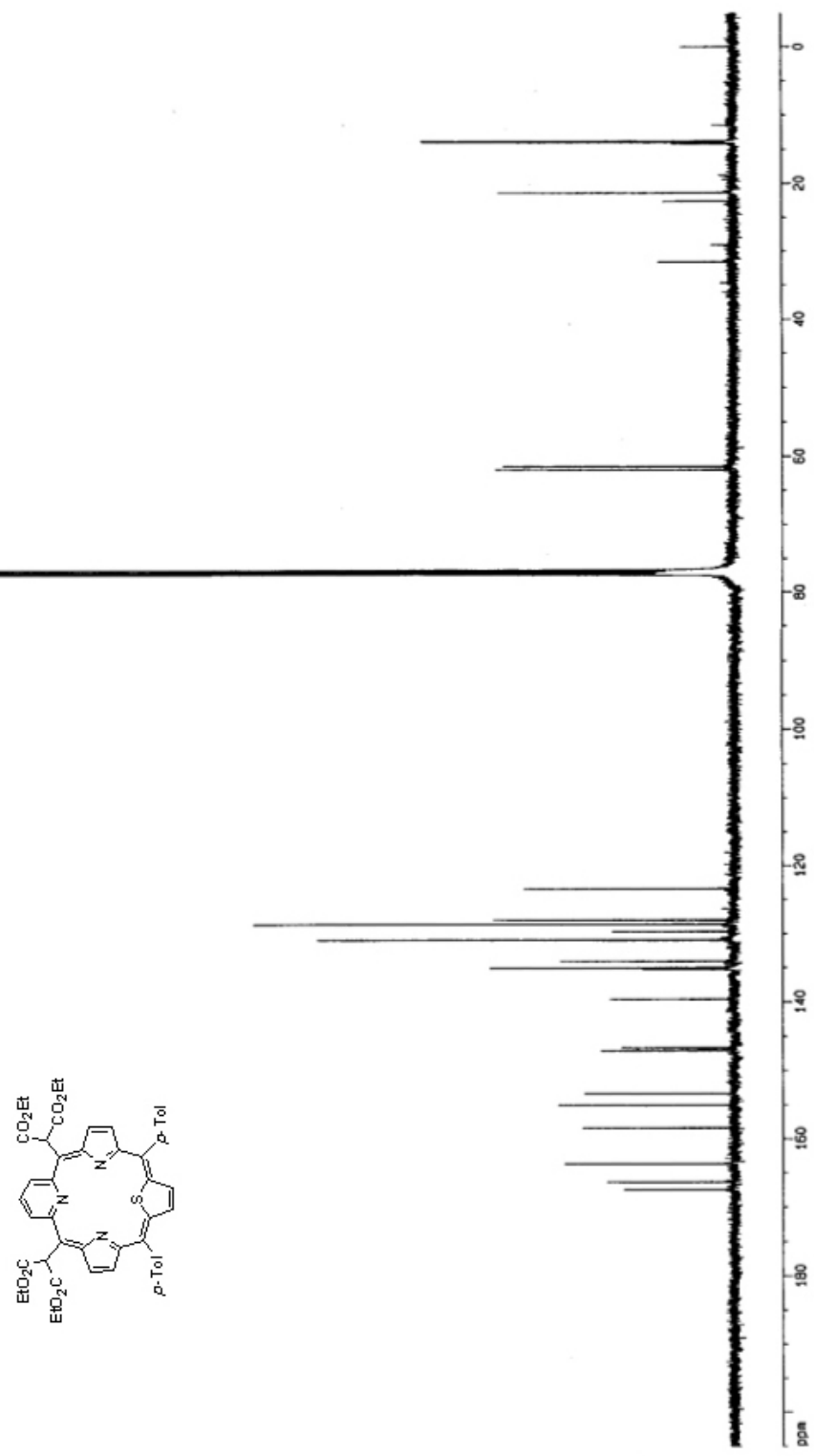
MALDI-TOF spectrum of 6,21-bis(diethylmalonyl)-11,16-di(p-tolyl)-24-thiapyriporphyrin 13 in $\mathrm{CH}_{2} \mathrm{Cl}_{2}$
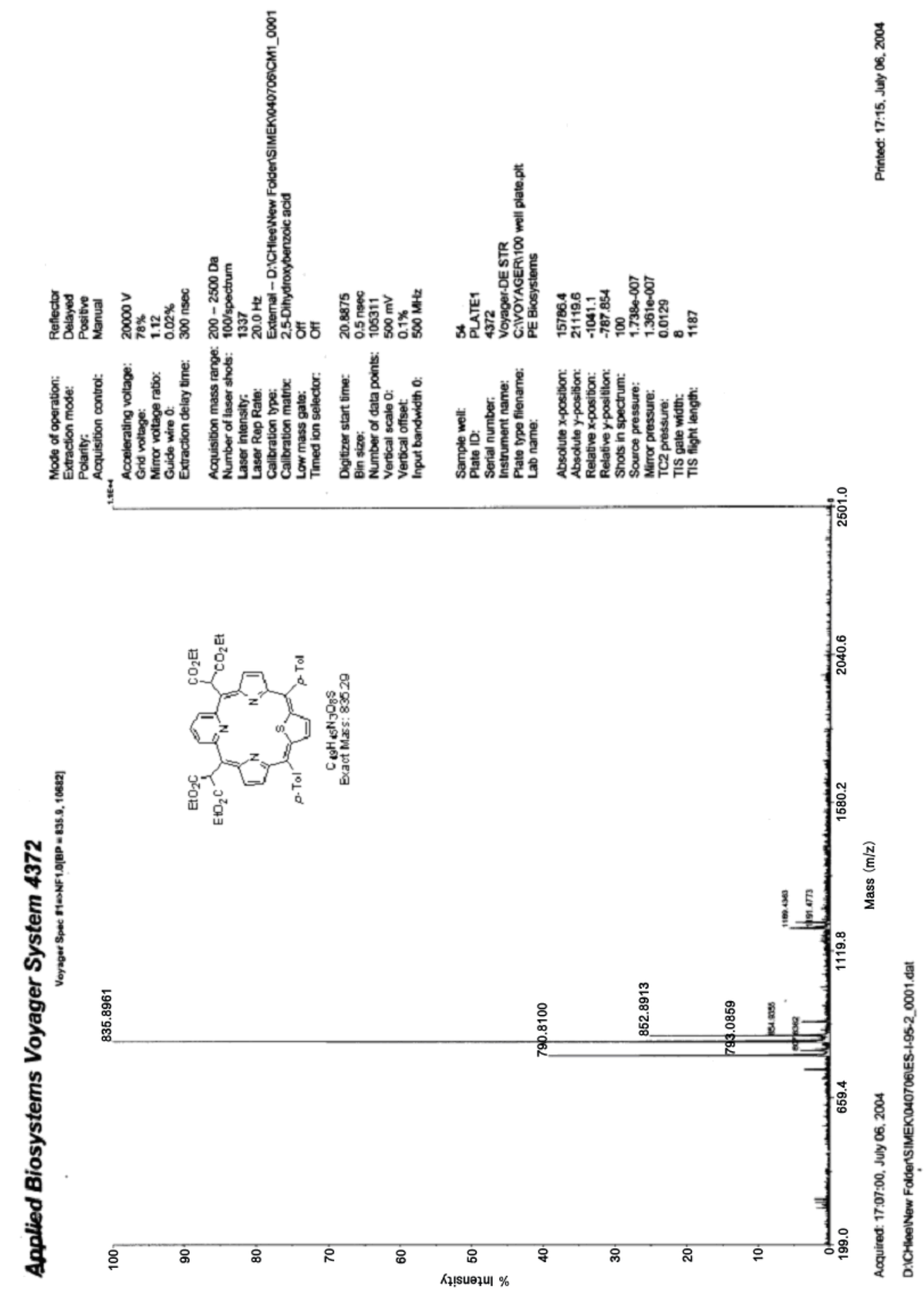


\section{Single crystal X-ray cryhstallographic data}

Crystal data, structure refinement, atomic coordinates, bond distance, bond angles, and anisotropic displacement parameters for $\mathbf{1 0 .}$

Figure S1. Intra- and intermolecular H-bonds for 10. Figure S2. Intermolecular C-H...O hydrogen bonds for $\mathbf{1 0 .}$

Figure S3. Intra- and intermolecular interactions for $\mathbf{1 0 .}$

Figure S4. Space-filling model of 10. Figure S5. Perspective view of core unit for $\mathbf{1 0 .}$

Crystal data, structure refinement, atomic coordinates, bond distance, bond angles, and anisotropic displacement parameters for 11. Figure S6. Intra- and intermolecular H-bonds for 11.

Figure S7. Intra- and intermolecular interactions between two adjacent molecules for $\mathbf{1 1}$.

Figure S8. Space-filling model of $\mathbf{1 1 .}$

Figure S9. Perspective view of core unit for 11. 
Table 1. Crystal data and structure refinement for $\mathbf{1 0 .}$

Empirical formula

Formula weight

Temperature

Wavelength

Crystal system

Space group

Unit cell dimensions

Volume

$\mathrm{Z}$

Density (calculated)

Absorption coefficient

$\mathrm{F}(000)$

Crystal size

Theta range for data collection

Index ranges

Reflections collected

Independent reflections

Completeness to theta $=28.26^{\circ}$

Refinement method

Data / restraints / parameters

Goodness-of-fit on $\mathrm{F}^{2}$

Final $\mathrm{R}$ indices $[\mathrm{I}>2 \operatorname{sigma}(\mathrm{I})]$

$\mathrm{R}$ indices (all data)

Largest diff. peak and hole
C48 H42 N2 O9

790.84

173(2) K

$0.71073 \AA$

Triclinic

P-1

$\mathrm{a}=12.5773(13) \AA \quad \square=103.581(2)^{\circ}$.

$\mathrm{b}=13.0527(14) \AA \quad \square=103.794(2)^{\circ}$.

$\mathrm{c}=14.9394(16) \AA \quad \square=113.814(2)^{\circ}$.

2022.1(4) $\AA^{3}$

2

$1.299 \mathrm{Mg} / \mathrm{m}^{3}$

$0.090 \mathrm{~mm}^{-1}$

832

$0.30 \times 0.20 \times 0.15 \mathrm{~mm}^{3}$

1.84 to $28.26^{\circ}$.

$-16<=\mathrm{h}<=14,-14<=\mathrm{k}<=16,-19<=\mathrm{l}<=19$

12886

$9026[\mathrm{R}($ int $)=0.0566]$

$90.1 \%$

Full-matrix least-squares on $\mathrm{F}^{2}$

9026 / 0 / 532

0.941

$\mathrm{R} 1=0.0574, \mathrm{wR} 2=0.1183$

$\mathrm{R} 1=0.1533, \mathrm{wR} 2=0.1526$

0.354 and -0.289 e. $\AA^{-3}$ 
Table 2. Atomic coordinates $\left(\times 10^{4}\right)$ and equivalent isotropic displacement parameters $\left(\AA^{2} \times 10^{3}\right)$ for 10. $U(e q)$ is defined as one third of the trace of the orthogonalized $U^{\mathrm{ij}}$ tensor.

\begin{tabular}{|c|c|c|c|c|}
\hline & $\mathrm{x}$ & $\mathrm{y}$ & $\mathrm{z}$ & $\mathrm{U}(\mathrm{eq})$ \\
\hline $\mathrm{O}(1)$ & $2330(2)$ & $8765(2)$ & 2971(1) & $27(1)$ \\
\hline $\mathrm{O}(2)$ & $5542(2)$ & $11960(2)$ & $1089(2)$ & $48(1)$ \\
\hline $\mathrm{O}(3)$ & 4511(2) & $12726(2)$ & $276(2)$ & $39(1)$ \\
\hline $\mathrm{O}(4)$ & $2653(2)$ & $9178(2)$ & $-1853(2)$ & $38(1)$ \\
\hline $\mathrm{O}(5)$ & $4661(2)$ & $10647(2)$ & $-1215(1)$ & $37(1)$ \\
\hline $\mathrm{O}(6)$ & $257(2)$ & $3674(2)$ & $-3012(2)$ & $52(1)$ \\
\hline $\mathrm{O}(7)$ & 1144(3) & $5677(2)$ & $-2442(2)$ & $61(1)$ \\
\hline $\mathrm{O}(8)$ & $84(2)$ & 2866(2) & $-1080(1)$ & $31(1)$ \\
\hline $\mathrm{O}(9)$ & $1877(2)$ & $3356(2)$ & $-1346(2)$ & $39(1)$ \\
\hline $\mathrm{N}(1)$ & $2107(2)$ & 9980(2) & $1613(2)$ & $26(1)$ \\
\hline $\mathrm{N}(2)$ & 2041(2) & $6638(2)$ & 1332(2) & $24(1)$ \\
\hline $\mathrm{C}(1)$ & 2093(3) & $10720(3)$ & $2427(2)$ & $25(1)$ \\
\hline $\mathrm{C}(2)$ & $2480(3)$ & 11837(3) & 2352(2) & $28(1)$ \\
\hline $\mathrm{C}(3)$ & $2770(3)$ & 11781(2) & $1506(2)$ & $26(1)$ \\
\hline $\mathrm{C}(4)$ & 2537(3) & $10621(2)$ & $1046(2)$ & $24(1)$ \\
\hline $\mathrm{C}(5)$ & 2617(3) & $10038(2)$ & $123(2)$ & $24(1)$ \\
\hline $\mathrm{C}(6)$ & 1662(3) & $8737(2)$ & $-381(2)$ & $23(1)$ \\
\hline $\mathrm{C}(7)$ & 411(3) & 8392(3) & $-847(2)$ & $28(1)$ \\
\hline $\mathrm{C}(8)$ & $-483(3)$ & 7185(3) & $-1236(2)$ & $31(1)$ \\
\hline $\mathrm{C}(9)$ & $-130(3)$ & 6329(3) & $-1135(2)$ & $27(1)$ \\
\hline $\mathrm{C}(10)$ & 1106(3) & 6667(2) & $-647(2)$ & $22(1)$ \\
\hline $\mathrm{C}(11)$ & 2016(3) & 7884(2) & $-271(2)$ & $24(1)$ \\
\hline$C(12)$ & $1439(3)$ & 5733(2) & $-497(2)$ & $24(1)$ \\
\hline$C(13)$ & 2092(3) & 5919(2) & $523(2)$ & $25(1)$ \\
\hline$C(14)$ & 2916(3) & $5527(3)$ & $884(2)$ & $29(1)$ \\
\hline$C(15)$ & $3331(3)$ & $5988(3)$ & 1915(2) & $31(1)$ \\
\hline$C(16)$ & 2770(3) & $6669(2)$ & 2192(2) & $26(1)$ \\
\hline$C(17)$ & 2841(3) & 7209(3) & 3183(2) & $27(1)$ \\
\hline$C(18)$ & $2540(3)$ & 8075(3) & $3510(2)$ & $27(1)$ \\
\hline $\mathrm{C}(19)$ & $2317(3)$ & $8433(3)$ & $4400(2)$ & $31(1)$ \\
\hline$C(20)$ & 1940(3) & $9246(3)$ & $4380(2)$ & $31(1)$ \\
\hline
\end{tabular}




\begin{tabular}{|c|c|c|c|c|}
\hline $\mathrm{C}(21)$ & 1984(3) & $9523(3)$ & $3512(2)$ & $27(1)$ \\
\hline $\mathrm{C}(22)$ & 1834(3) & $10389(3)$ & $3243(2)$ & $28(1)$ \\
\hline$C(23)$ & $3530(3)$ & $10614(3)$ & $-189(2)$ & $27(1)$ \\
\hline$C(24)$ & $4647(3)$ & $11826(3)$ & $480(2)$ & $33(1)$ \\
\hline$C(25)$ & $5587(3)$ & 13931(3) & $869(3)$ & $53(1)$ \\
\hline$C(26)$ & $5585(4)$ & $14465(3)$ & $1868(3)$ & $70(1)$ \\
\hline$C(27)$ & $3531(3)$ & $10042(3)$ & $-1180(2)$ & $29(1)$ \\
\hline $\mathrm{C}(28)$ & $4826(3)$ & $10158(3)$ & $-2126(2)$ & $44(1)$ \\
\hline $\mathrm{C}(29)$ & $4303(3)$ & $10505(3)$ & $-2928(2)$ & $52(1)$ \\
\hline$C(30)$ & $1105(3)$ & $4736(2)$ & $-1285(2)$ & $25(1)$ \\
\hline $\mathrm{C}(31)$ & $766(3)$ & $4625(3)$ & $-2335(2)$ & $30(1)$ \\
\hline$C(32)$ & $776(4)$ & $5688(4)$ & $-3440(3)$ & $72(1)$ \\
\hline$C(33)$ & 1890(4) & $6248(5)$ & $-3646(3)$ & $94(2)$ \\
\hline$C(34)$ & $970(3)$ & $3568(3)$ & $-1205(2)$ & $27(1)$ \\
\hline$C(35)$ & 1706(3) & $2155(3)$ & $-1463(3)$ & $44(1)$ \\
\hline$C(36)$ & 2463(4) & 1931(3) & $-2036(3)$ & $57(1)$ \\
\hline$C(37)$ & $3270(3)$ & $6724(3)$ & $3922(2)$ & $30(1)$ \\
\hline$C(38)$ & $2660(3)$ & $5501(3)$ & $3759(2)$ & $39(1)$ \\
\hline $\mathrm{C}(39)$ & $3080(4)$ & $5064(3)$ & $4446(3)$ & $50(1)$ \\
\hline$C(40)$ & 4107(4) & $5837(4)$ & $5304(3)$ & $56(1)$ \\
\hline $\mathrm{C}(41)$ & $4718(3)$ & 7054(4) & $5489(3)$ & $57(1)$ \\
\hline $\mathrm{C}(42)$ & 4307(3) & $7503(3)$ & $4802(2)$ & $43(1)$ \\
\hline$C(43)$ & 1453(3) & $11154(3)$ & $3860(2)$ & $30(1)$ \\
\hline $\mathrm{C}(44)$ & 2151(3) & 11844(3) & $4867(2)$ & $38(1)$ \\
\hline $\mathrm{C}(45)$ & $1723(4)$ & $12507(3)$ & $5410(2)$ & $51(1)$ \\
\hline$C(46)$ & $618(4)$ & $12485(3)$ & 4953(3) & $50(1)$ \\
\hline $\mathrm{C}(47)$ & $-59(3)$ & 11827(3) & $3958(3)$ & $48(1)$ \\
\hline $\mathrm{C}(48)$ & $364(3)$ & $11179(3)$ & $3414(2)$ & $38(1)$ \\
\hline
\end{tabular}


Table 3. Bond lengths $[\AA]$ and angles $\left[{ }^{\circ}\right]$ for $\mathbf{1 0}$.

\begin{tabular}{|c|c|}
\hline $\mathrm{O}(1)-\mathrm{C}(18)$ & $1.399(3)$ \\
\hline $\mathrm{O}(1)-\mathrm{C}(21)$ & $1.401(3)$ \\
\hline $\mathrm{O}(2)-\mathrm{C}(24)$ & $1.184(4)$ \\
\hline $\mathrm{O}(3)-\mathrm{C}(24)$ & $1.345(4)$ \\
\hline $\mathrm{O}(3)-\mathrm{C}(25)$ & $1.465(4)$ \\
\hline $\mathrm{O}(4)-\mathrm{C}(27)$ & $1.198(3)$ \\
\hline $\mathrm{O}(5)-\mathrm{C}(27)$ & $1.339(3)$ \\
\hline $\mathrm{O}(5)-\mathrm{C}(28)$ & $1.462(3)$ \\
\hline $\mathrm{O}(6)-\mathrm{C}(31)$ & $1.197(3)$ \\
\hline $\mathrm{O}(7)-\mathrm{C}(31)$ & $1.323(3)$ \\
\hline $\mathrm{O}(7)-\mathrm{C}(32)$ & $1.458(4)$ \\
\hline $\mathrm{O}(8)-\mathrm{C}(34)$ & $1.213(3)$ \\
\hline $\mathrm{O}(9)-\mathrm{C}(34)$ & $1.329(3)$ \\
\hline $\mathrm{O}(9)-\mathrm{C}(35)$ & $1.455(3)$ \\
\hline $\mathrm{N}(1)-\mathrm{C}(1)$ & $1.373(3)$ \\
\hline $\mathrm{N}(1)-\mathrm{C}(4)$ & $1.376(3)$ \\
\hline $\mathrm{N}(2)-\mathrm{C}(16)$ & $1.370(3)$ \\
\hline $\mathrm{N}(2)-\mathrm{C}(13)$ & $1.378(3)$ \\
\hline $\mathrm{C}(1)-\mathrm{C}(2)$ & $1.381(4)$ \\
\hline $\mathrm{C}(1)-\mathrm{C}(22)$ & $1.450(4)$ \\
\hline $\mathrm{C}(2)-\mathrm{C}(3)$ & $1.391(4)$ \\
\hline $\mathrm{C}(3)-\mathrm{C}(4)$ & $1.381(4)$ \\
\hline$C(4)-C(5)$ & $1.458(4)$ \\
\hline $\mathrm{C}(5)-\mathrm{C}(23)$ & $1.351(4)$ \\
\hline$C(5)-C(6)$ & $1.488(4)$ \\
\hline$C(6)-C(11)$ & $1.385(4)$ \\
\hline$C(6)-C(7)$ & $1.386(4)$ \\
\hline$C(7)-C(8)$ & $1.382(4)$ \\
\hline $\mathrm{C}(8)-\mathrm{C}(9)$ & $1.384(4)$ \\
\hline$C(9)-C(10)$ & $1.377(4)$ \\
\hline$C(10)-C(11)$ & $1.398(4)$ \\
\hline$C(10)-C(12)$ & $1.486(4)$ \\
\hline$C(12)-C(30)$ & $1.364(4)$ \\
\hline $\mathrm{C}(12)-\mathrm{C}(13)$ & $1.457(4)$ \\
\hline
\end{tabular}




\begin{tabular}{|c|c|}
\hline$C(13)-C(14)$ & $1.384(4)$ \\
\hline$C(14)-C(15)$ & $1.390(4)$ \\
\hline$C(15)-C(16)$ & $1.389(4)$ \\
\hline $\mathrm{C}(16)-\mathrm{C}(17)$ & $1.447(4)$ \\
\hline $\mathrm{C}(17)-\mathrm{C}(18)$ & $1.355(4)$ \\
\hline $\mathrm{C}(17)-\mathrm{C}(37)$ & $1.494(4)$ \\
\hline$C(18)-C(19)$ & $1.433(4)$ \\
\hline$C(19)-C(20)$ & $1.330(4)$ \\
\hline$C(20)-C(21)$ & $1.433(4)$ \\
\hline$C(21)-C(22)$ & $1.349(4)$ \\
\hline$C(22)-C(43)$ & $1.496(4)$ \\
\hline $\mathrm{C}(23)-\mathrm{C}(27)$ & $1.494(4)$ \\
\hline$C(23)-C(24)$ & $1.506(4)$ \\
\hline$C(25)-C(26)$ & $1.493(4)$ \\
\hline$C(28)-C(29)$ & $1.481(4)$ \\
\hline $\mathrm{C}(30)-\mathrm{C}(31)$ & $1.478(4)$ \\
\hline$C(30)-C(34)$ & $1.501(4)$ \\
\hline$C(32)-C(33)$ & $1.441(5)$ \\
\hline$C(35)-C(36)$ & $1.489(4)$ \\
\hline $\mathrm{C}(37)-\mathrm{C}(38)$ & $1.391(4)$ \\
\hline$C(37)-C(42)$ & $1.395(4)$ \\
\hline $\mathrm{C}(38)-\mathrm{C}(39)$ & $1.385(4)$ \\
\hline $\mathrm{C}(39)-\mathrm{C}(40)$ & $1.371(5)$ \\
\hline $\mathrm{C}(40)-\mathrm{C}(41)$ & $1.378(5)$ \\
\hline $\mathrm{C}(41)-\mathrm{C}(42)$ & $1.393(5)$ \\
\hline $\mathrm{C}(43)-\mathrm{C}(48)$ & $1.391(4)$ \\
\hline $\mathrm{C}(43)-\mathrm{C}(44)$ & $1.393(4)$ \\
\hline $\mathrm{C}(44)-\mathrm{C}(45)$ & $1.398(4)$ \\
\hline $\mathrm{C}(45)-\mathrm{C}(46)$ & $1.380(5)$ \\
\hline $\mathrm{C}(46)-\mathrm{C}(47)$ & $1.369(5)$ \\
\hline $\mathrm{C}(47)-\mathrm{C}(48)$ & $1.380(4)$ \\
\hline $\mathrm{C}(18)-\mathrm{O}(1)-\mathrm{C}(21)$ & $107.9(2)$ \\
\hline $\mathrm{C}(24)-\mathrm{O}(3)-\mathrm{C}(25)$ & $114.5(3)$ \\
\hline $\mathrm{C}(27)-\mathrm{O}(5)-\mathrm{C}(28)$ & $116.4(2)$ \\
\hline $\mathrm{C}(31)-\mathrm{O}(7)-\mathrm{C}(32)$ & $118.7(3)$ \\
\hline
\end{tabular}




\begin{tabular}{|c|c|}
\hline $\mathrm{C}(34)-\mathrm{O}(9)-\mathrm{C}(35)$ & $116.8(2)$ \\
\hline $\mathrm{C}(1)-\mathrm{N}(1)-\mathrm{C}(4)$ & $109.9(2)$ \\
\hline $\mathrm{C}(16)-\mathrm{N}(2)-\mathrm{C}(13)$ & $110.0(2)$ \\
\hline $\mathrm{N}(1)-\mathrm{C}(1)-\mathrm{C}(2)$ & $106.9(2)$ \\
\hline $\mathrm{N}(1)-\mathrm{C}(1)-\mathrm{C}(22)$ & $125.0(2)$ \\
\hline $\mathrm{C}(2)-\mathrm{C}(1)-\mathrm{C}(22)$ & $127.8(2)$ \\
\hline $\mathrm{C}(1)-\mathrm{C}(2)-\mathrm{C}(3)$ & $108.2(2)$ \\
\hline $\mathrm{C}(4)-\mathrm{C}(3)-\mathrm{C}(2)$ & $108.1(2)$ \\
\hline $\mathrm{N}(1)-\mathrm{C}(4)-\mathrm{C}(3)$ & $106.8(2)$ \\
\hline $\mathrm{N}(1)-\mathrm{C}(4)-\mathrm{C}(5)$ & 121.1(2) \\
\hline$C(3)-C(4)-C(5)$ & $132.0(2)$ \\
\hline$C(23)-C(5)-C(4)$ & $122.5(2)$ \\
\hline$C(23)-C(5)-C(6)$ & $124.3(2)$ \\
\hline $\mathrm{C}(4)-\mathrm{C}(5)-\mathrm{C}(6)$ & $113.0(2)$ \\
\hline $\mathrm{C}(11)-\mathrm{C}(6)-\mathrm{C}(7)$ & $120.3(3)$ \\
\hline$C(11)-C(6)-C(5)$ & $118.8(3)$ \\
\hline$C(7)-C(6)-C(5)$ & $120.4(3)$ \\
\hline $\mathrm{C}(8)-\mathrm{C}(7)-\mathrm{C}(6)$ & $119.9(3)$ \\
\hline $\mathrm{C}(7)-\mathrm{C}(8)-\mathrm{C}(9)$ & $119.8(3)$ \\
\hline $\mathrm{C}(10)-\mathrm{C}(9)-\mathrm{C}(8)$ & $120.7(3)$ \\
\hline $\mathrm{C}(9)-\mathrm{C}(10)-\mathrm{C}(11)$ & $119.6(3)$ \\
\hline $\mathrm{C}(9)-\mathrm{C}(10)-\mathrm{C}(12)$ & $119.2(2)$ \\
\hline $\mathrm{C}(11)-\mathrm{C}(10)-\mathrm{C}(12)$ & $121.2(3)$ \\
\hline $\mathrm{C}(6)-\mathrm{C}(11)-\mathrm{C}(10)$ & $119.6(3)$ \\
\hline $\mathrm{C}(30)-\mathrm{C}(12)-\mathrm{C}(13)$ & $122.5(3)$ \\
\hline $\mathrm{C}(30)-\mathrm{C}(12)-\mathrm{C}(10)$ & $120.6(3)$ \\
\hline $\mathrm{C}(13)-\mathrm{C}(12)-\mathrm{C}(10)$ & $116.9(2)$ \\
\hline $\mathrm{N}(2)-\mathrm{C}(13)-\mathrm{C}(14)$ & 107.2(2) \\
\hline $\mathrm{N}(2)-\mathrm{C}(13)-\mathrm{C}(12)$ & $123.0(2)$ \\
\hline $\mathrm{C}(14)-\mathrm{C}(13)-\mathrm{C}(12)$ & $129.7(2)$ \\
\hline $\mathrm{C}(13)-\mathrm{C}(14)-\mathrm{C}(15)$ & $107.6(2)$ \\
\hline $\mathrm{C}(16)-\mathrm{C}(15)-\mathrm{C}(14)$ & $108.5(3)$ \\
\hline $\mathrm{N}(2)-\mathrm{C}(16)-\mathrm{C}(15)$ & $106.6(2)$ \\
\hline $\mathrm{N}(2)-\mathrm{C}(16)-\mathrm{C}(17)$ & $126.2(2)$ \\
\hline $\mathrm{C}(15)-\mathrm{C}(16)-\mathrm{C}(17)$ & $126.9(3)$ \\
\hline $\mathrm{C}(18)-\mathrm{C}(17)-\mathrm{C}(16)$ & $127.9(3)$ \\
\hline
\end{tabular}




\begin{tabular}{|c|c|}
\hline $\mathrm{C}(18)-\mathrm{C}(17)-\mathrm{C}(37)$ & $116.6(2)$ \\
\hline $\mathrm{C}(16)-\mathrm{C}(17)-\mathrm{C}(37)$ & $115.5(2)$ \\
\hline $\mathrm{C}(17)-\mathrm{C}(18)-\mathrm{O}(1)$ & $123.6(2)$ \\
\hline $\mathrm{C}(17)-\mathrm{C}(18)-\mathrm{C}(19)$ & $129.3(3)$ \\
\hline $\mathrm{O}(1)-\mathrm{C}(18)-\mathrm{C}(19)$ & $107.0(2)$ \\
\hline C(20)-C(19)-C(18) & $109.0(3)$ \\
\hline$C(19)-C(20)-C(21)$ & $109.0(2)$ \\
\hline $\mathrm{C}(22)-\mathrm{C}(21)-\mathrm{O}(1)$ & $122.8(2)$ \\
\hline $\mathrm{C}(22)-\mathrm{C}(21)-\mathrm{C}(20)$ & 130.1(3) \\
\hline $\mathrm{O}(1)-\mathrm{C}(21)-\mathrm{C}(20)$ & $106.9(2)$ \\
\hline $\mathrm{C}(21)-\mathrm{C}(22)-\mathrm{C}(1)$ & $125.7(2)$ \\
\hline $\mathrm{C}(21)-\mathrm{C}(22)-\mathrm{C}(43)$ & $119.3(2)$ \\
\hline $\mathrm{C}(1)-\mathrm{C}(22)-\mathrm{C}(43)$ & $114.9(2)$ \\
\hline $\mathrm{C}(5)-\mathrm{C}(23)-\mathrm{C}(27)$ & $121.7(3)$ \\
\hline $\mathrm{C}(5)-\mathrm{C}(23)-\mathrm{C}(24)$ & $121.2(2)$ \\
\hline C(27)-C(23)-C(24) & $116.9(2)$ \\
\hline $\mathrm{O}(2)-\mathrm{C}(24)-\mathrm{O}(3)$ & $124.7(3)$ \\
\hline $\mathrm{O}(2)-\mathrm{C}(24)-\mathrm{C}(23)$ & $124.0(3)$ \\
\hline $\mathrm{O}(3)-\mathrm{C}(24)-\mathrm{C}(23)$ & $111.3(3)$ \\
\hline $\mathrm{O}(3)-\mathrm{C}(25)-\mathrm{C}(26)$ & 113.1(3) \\
\hline $\mathrm{O}(4)-\mathrm{C}(27)-\mathrm{O}(5)$ & $124.5(3)$ \\
\hline $\mathrm{O}(4)-\mathrm{C}(27)-\mathrm{C}(23)$ & $125.5(3)$ \\
\hline $\mathrm{O}(5)-\mathrm{C}(27)-\mathrm{C}(23)$ & $110.0(2)$ \\
\hline $\mathrm{O}(5)-\mathrm{C}(28)-\mathrm{C}(29)$ & $112.0(3)$ \\
\hline $\mathrm{C}(12)-\mathrm{C}(30)-\mathrm{C}(31)$ & $125.5(3)$ \\
\hline $\mathrm{C}(12)-\mathrm{C}(30)-\mathrm{C}(34)$ & $124.0(3)$ \\
\hline $\mathrm{C}(31)-\mathrm{C}(30)-\mathrm{C}(34)$ & $110.5(2)$ \\
\hline $\mathrm{O}(6)-\mathrm{C}(31)-\mathrm{O}(7)$ & $124.0(3)$ \\
\hline $\mathrm{O}(6)-\mathrm{C}(31)-\mathrm{C}(30)$ & $122.6(3)$ \\
\hline $\mathrm{O}(7)-\mathrm{C}(31)-\mathrm{C}(30)$ & $113.4(2)$ \\
\hline $\mathrm{C}(33)-\mathrm{C}(32)-\mathrm{O}(7)$ & $108.6(4)$ \\
\hline $\mathrm{O}(8)-\mathrm{C}(34)-\mathrm{O}(9)$ & $123.9(3)$ \\
\hline $\mathrm{O}(8)-\mathrm{C}(34)-\mathrm{C}(30)$ & $123.0(3)$ \\
\hline $\mathrm{O}(9)-\mathrm{C}(34)-\mathrm{C}(30)$ & $112.9(2)$ \\
\hline $\mathrm{O}(9)-\mathrm{C}(35)-\mathrm{C}(36)$ & $107.4(3)$ \\
\hline $\mathrm{C}(38)-\mathrm{C}(37)-\mathrm{C}(42)$ & $118.4(3)$ \\
\hline
\end{tabular}




$\begin{array}{ll}\mathrm{C}(38)-\mathrm{C}(37)-\mathrm{C}(17) & 121.6(3) \\ \mathrm{C}(42)-\mathrm{C}(37)-\mathrm{C}(17) & 120.0(3) \\ \mathrm{C}(39)-\mathrm{C}(38)-\mathrm{C}(37) & 120.9(3) \\ \mathrm{C}(40)-\mathrm{C}(39)-\mathrm{C}(38) & 120.2(4) \\ \mathrm{C}(39)-\mathrm{C}(40)-\mathrm{C}(41) & 120.1(3) \\ \mathrm{C}(40)-\mathrm{C}(41)-\mathrm{C}(42) & 120.2(4) \\ \mathrm{C}(41)-\mathrm{C}(42)-\mathrm{C}(37) & 120.2(3) \\ \mathrm{C}(48)-\mathrm{C}(43)-\mathrm{C}(44) & 118.6(3) \\ \mathrm{C}(48)-\mathrm{C}(43)-\mathrm{C}(22) & 119.0(3) \\ \mathrm{C}(44)-\mathrm{C}(43)-\mathrm{C}(22) & 122.4(3) \\ \mathrm{C}(43)-\mathrm{C}(44)-\mathrm{C}(45) & 119.6(3) \\ \mathrm{C}(46)-\mathrm{C}(45)-\mathrm{C}(44) & 120.3(3) \\ \mathrm{C}(47)-\mathrm{C}(46)-\mathrm{C}(45) & 120.3(3) \\ \mathrm{C}(46)-\mathrm{C}(47)-\mathrm{C}(48) & 119.8(3) \\ \mathrm{C}(47)-\mathrm{C}(48)-\mathrm{C}(43) & 121.3(3)\end{array}$

Symmetry transformations used to generate equivalent atoms: 
Table 4. Anisotropic displacement parameters $\left(\AA^{2} \times 10^{3}\right)$ for 10. The anisotropic displacement factor exponent takes the form: $-2 \square^{2}\left[h^{2} a^{* 2} U^{11}+\ldots+2 h k a^{*} b^{*} U^{12}\right]$

\begin{tabular}{|c|c|c|c|c|c|c|}
\hline & $\mathrm{U}^{11}$ & $\mathrm{U}^{22}$ & $\mathrm{U}^{33}$ & $\mathrm{U}^{23}$ & $\mathrm{U}^{13}$ & $\mathrm{U}^{12}$ \\
\hline $\mathrm{O}(1)$ & $40(1)$ & $31(1)$ & $22(1)$ & $12(1)$ & $17(1)$ & $23(1)$ \\
\hline $\mathrm{O}(2)$ & $40(2)$ & $48(2)$ & $44(2)$ & 13(1) & $5(1)$ & 19(1) \\
\hline $\mathrm{O}(3)$ & $43(1)$ & $28(1)$ & $45(1)$ & $13(1)$ & 21(1) & $15(1)$ \\
\hline $\mathrm{O}(4)$ & $45(1)$ & $38(1)$ & $27(1)$ & $9(1)$ & $18(1)$ & $13(1)$ \\
\hline $\mathrm{O}(5)$ & $38(1)$ & $44(1)$ & $27(1)$ & $9(1)$ & $22(1)$ & $16(1)$ \\
\hline $\mathrm{O}(6)$ & $76(2)$ & $34(1)$ & $29(1)$ & $2(1)$ & $20(1)$ & $17(1)$ \\
\hline $\mathrm{O}(7)$ & $118(2)$ & $38(2)$ & $30(1)$ & $20(1)$ & $33(2)$ & $36(2)$ \\
\hline $\mathrm{O}(8)$ & $37(1)$ & $26(1)$ & $34(1)$ & 11(1) & $16(1)$ & $18(1)$ \\
\hline $\mathrm{O}(9)$ & $38(1)$ & $30(1)$ & $57(2)$ & 14(1) & 21(1) & $23(1)$ \\
\hline $\mathrm{N}(1)$ & $38(2)$ & $23(1)$ & $25(1)$ & $10(1)$ & $18(1)$ & $16(1)$ \\
\hline $\mathrm{N}(2)$ & $28(1)$ & $25(1)$ & $22(1)$ & $8(1)$ & $9(1)$ & $18(1)$ \\
\hline $\mathrm{C}(1)$ & $30(2)$ & $29(2)$ & $22(2)$ & $8(1)$ & $12(1)$ & 19(1) \\
\hline $\mathrm{C}(2)$ & $33(2)$ & $28(2)$ & $26(2)$ & $8(1)$ & 12(1) & 19(1) \\
\hline $\mathrm{C}(3)$ & $31(2)$ & $24(2)$ & $29(2)$ & $12(1)$ & 16(1) & $15(1)$ \\
\hline $\mathrm{C}(4)$ & $32(2)$ & $23(2)$ & $23(2)$ & $12(1)$ & $15(1)$ & $14(1)$ \\
\hline$C(5)$ & $33(2)$ & $22(2)$ & $24(2)$ & 11(1) & $13(1)$ & $17(1)$ \\
\hline $\mathrm{C}(6)$ & $29(2)$ & $23(2)$ & $18(2)$ & $7(1)$ & $14(1)$ & $13(1)$ \\
\hline $\mathrm{C}(7)$ & $37(2)$ & $28(2)$ & $29(2)$ & $16(1)$ & $15(2)$ & $21(2)$ \\
\hline $\mathrm{C}(8)$ & $26(2)$ & $34(2)$ & $31(2)$ & $12(1)$ & $5(1)$ & $16(2)$ \\
\hline $\mathrm{C}(9)$ & $28(2)$ & $21(2)$ & $26(2)$ & $7(1)$ & $8(1)$ & $9(1)$ \\
\hline $\mathrm{C}(10)$ & $27(2)$ & $25(2)$ & $16(1)$ & $7(1)$ & $10(1)$ & $14(1)$ \\
\hline $\mathrm{C}(11)$ & $29(2)$ & $29(2)$ & $17(2)$ & $7(1)$ & 12(1) & $16(1)$ \\
\hline$C(12)$ & $27(2)$ & $23(2)$ & $26(2)$ & $9(1)$ & $13(1)$ & $13(1)$ \\
\hline$C(13)$ & $27(2)$ & $24(2)$ & $24(2)$ & $7(1)$ & $12(1)$ & $13(1)$ \\
\hline$C(14)$ & $31(2)$ & $35(2)$ & $28(2)$ & $9(1)$ & $13(2)$ & $23(2)$ \\
\hline$C(15)$ & $33(2)$ & $38(2)$ & $29(2)$ & $14(2)$ & $10(2)$ & $23(2)$ \\
\hline$C(16)$ & $22(2)$ & $28(2)$ & $25(2)$ & 10(1) & $7(1)$ & $12(1)$ \\
\hline $\mathrm{C}(17)$ & $30(2)$ & $30(2)$ & $22(2)$ & $10(1)$ & $9(1)$ & $17(1)$ \\
\hline$C(18)$ & $32(2)$ & $31(2)$ & $22(2)$ & $13(1)$ & 10(1) & $15(1)$ \\
\hline$C(19)$ & $45(2)$ & $31(2)$ & $20(2)$ & 11(1) & $14(2)$ & $20(2)$ \\
\hline$C(20)$ & $47(2)$ & $30(2)$ & $24(2)$ & $10(1)$ & $20(2)$ & $21(2)$ \\
\hline
\end{tabular}




\begin{tabular}{lllllll}
$\mathrm{C}(21)$ & $36(2)$ & $28(2)$ & $22(2)$ & $8(1)$ & $15(1)$ & $19(2)$ \\
$\mathrm{C}(22)$ & $34(2)$ & $29(2)$ & $23(2)$ & $8(1)$ & $16(1)$ & $16(1)$ \\
$\mathrm{C}(23)$ & $34(2)$ & $26(2)$ & $28(2)$ & $11(1)$ & $17(2)$ & $17(1)$ \\
$\mathrm{C}(24)$ & $42(2)$ & $31(2)$ & $36(2)$ & $15(2)$ & $27(2)$ & $20(2)$ \\
$\mathrm{C}(25)$ & $50(2)$ & $30(2)$ & $64(3)$ & $12(2)$ & $24(2)$ & $7(2)$ \\
$\mathrm{C}(26)$ & $64(3)$ & $45(2)$ & $69(3)$ & $-5(2)$ & $29(2)$ & $11(2)$ \\
$\mathrm{C}(27)$ & $37(2)$ & $28(2)$ & $27(2)$ & $11(1)$ & $16(2)$ & $18(2)$ \\
$\mathrm{C}(28)$ & $44(2)$ & $58(2)$ & $31(2)$ & $10(2)$ & $23(2)$ & $25(2)$ \\
$\mathrm{C}(29)$ & $54(2)$ & $67(3)$ & $36(2)$ & $23(2)$ & $26(2)$ & $23(2)$ \\
$\mathrm{C}(30)$ & $26(2)$ & $25(2)$ & $26(2)$ & $9(1)$ & $13(1)$ & $15(1)$ \\
$\mathrm{C}(31)$ & $33(2)$ & $32(2)$ & $28(2)$ & $9(2)$ & $15(2)$ & $17(2)$ \\
$\mathrm{C}(32)$ & $118(4)$ & $80(3)$ & $41(2)$ & $42(2)$ & $38(3)$ & $54(3)$ \\
$\mathrm{C}(33)$ & $107(4)$ & $141(5)$ & $62(3)$ & $54(3)$ & $53(3)$ & $65(4)$ \\
$\mathrm{C}(34)$ & $28(2)$ & $30(2)$ & $22(2)$ & $5(1)$ & $9(1)$ & $16(2)$ \\
$\mathrm{C}(35)$ & $54(2)$ & $32(2)$ & $62(2)$ & $20(2)$ & $28(2)$ & $31(2)$ \\
$\mathrm{C}(36)$ & $83(3)$ & $53(2)$ & $61(3)$ & $22(2)$ & $35(2)$ & $52(2)$ \\
$\mathrm{C}(37)$ & $37(2)$ & $37(2)$ & $23(2)$ & $13(1)$ & $13(2)$ & $22(2)$ \\
$\mathrm{C}(38)$ & $56(2)$ & $40(2)$ & $30(2)$ & $15(2)$ & $17(2)$ & $29(2)$ \\
$\mathrm{C}(39)$ & $86(3)$ & $55(2)$ & $42(2)$ & $31(2)$ & $37(2)$ & $50(2)$ \\
$\mathrm{C}(40)$ & $78(3)$ & $86(3)$ & $45(2)$ & $44(2)$ & $31(2)$ & $61(3)$ \\
$\mathrm{C}(41)$ & $50(2)$ & $84(3)$ & $33(2)$ & $27(2)$ & $4(2)$ & $32(2)$ \\
$\mathrm{C}(42)$ & $43(2)$ & $51(2)$ & $32(2)$ & $17(2)$ & $6(2)$ & $22(2)$ \\
$\mathrm{C}(43)$ & $45(2)$ & $29(2)$ & $26(2)$ & $14(1)$ & $22(2)$ & $20(2)$ \\
$\mathrm{C}(44)$ & $59(2)$ & $35(2)$ & $26(2)$ & $12(2)$ & $19(2)$ & $25(2)$ \\
$\mathrm{C}(45)$ & $91(3)$ & $41(2)$ & $31(2)$ & $15(2)$ & $33(2)$ & $35(2)$ \\
$\mathrm{C}(46)$ & $76(3)$ & $42(2)$ & $59(3)$ & $23(2)$ & $48(2)$ & $38(2)$ \\
$\mathrm{C}(47)$ & $54(2)$ & $43(2)$ & $63(3)$ & $20(2)$ & $38(2)$ & $29(2)$ \\
$\mathrm{C}(48)$ & $43(2)$ & $33(2)$ & $42(2)$ & $12(2)$ & $22(2)$ & $20(2)$ \\
\hline & & & & & & \\
\hline
\end{tabular}


Table 5. Hydrogen coordinates ( x 10 $0^{4}$ ) and isotropic displacement parameters $\left(\AA^{2} \mathrm{x} 10^{3}\right)$ for 10.

\begin{tabular}{|c|c|c|c|c|}
\hline & $\mathrm{x}$ & $\mathrm{y}$ & $\mathrm{z}$ & $\mathrm{U}(\mathrm{eq})$ \\
\hline $\mathrm{H}(1 \mathrm{~A})$ & 1875 & 9210 & 1473 & 32 \\
\hline $\mathrm{H}(2 \mathrm{~A})$ & 1604 & 7021 & 1301 & 28 \\
\hline $\mathrm{H}(2 \mathrm{~B})$ & 2538 & 12525 & 2801 & 33 \\
\hline $\mathrm{H}(3 \mathrm{~A})$ & 3075 & 12428 & 1283 & 31 \\
\hline $\mathrm{H}(7 \mathrm{~A})$ & 168 & 8983 & -898 & 34 \\
\hline $\mathrm{H}(8 \mathrm{~A})$ & -1338 & 6944 & -1573 & 37 \\
\hline $\mathrm{H}(9 \mathrm{~A})$ & -746 & 5501 & -1404 & 32 \\
\hline $\mathrm{H}(11 \mathrm{~A})$ & 2873 & 8124 & 57 & 29 \\
\hline $\mathrm{H}(14 \mathrm{~A})$ & 3154 & 5033 & 498 & 35 \\
\hline $\mathrm{H}(15 \mathrm{~A})$ & 3902 & 5859 & 2356 & 38 \\
\hline H(19A) & 2421 & 8136 & 4919 & 37 \\
\hline $\mathrm{H}(20 \mathrm{~A})$ & 1683 & 9589 & 4864 & 37 \\
\hline $\mathrm{H}(25 \mathrm{~A})$ & 5584 & 14472 & 503 & 64 \\
\hline $\mathrm{H}(25 \mathrm{~B})$ & 6368 & 13883 & 954 & 64 \\
\hline $\mathrm{H}(26 \mathrm{~A})$ & 6321 & 15269 & 2228 & 83 \\
\hline $\mathrm{H}(26 \mathrm{~B})$ & 5613 & 13947 & 2243 & 83 \\
\hline $\mathrm{H}(26 \mathrm{C})$ & 4821 & 14530 & 1790 & 83 \\
\hline $\mathrm{H}(28 \mathrm{~A})$ & 4406 & 9267 & -2352 & 52 \\
\hline $\mathrm{H}(28 \mathrm{~B})$ & 5731 & 10457 & -1985 & 52 \\
\hline H(29A) & 4432 & 10161 & -3525 & 63 \\
\hline $\mathrm{H}(29 \mathrm{~B})$ & 4729 & 11387 & -2713 & 63 \\
\hline $\mathrm{H}(29 \mathrm{C})$ & 3404 & 10198 & -3079 & 63 \\
\hline $\mathrm{H}(32 \mathrm{~A})$ & 220 & 4852 & -3931 & 86 \\
\hline $\mathrm{H}(32 \mathrm{~B})$ & 313 & 6145 & -3488 & 86 \\
\hline $\mathrm{H}(33 \mathrm{~A})$ & 1656 & 6261 & -4316 & 113 \\
\hline $\mathrm{H}(33 \mathrm{~B})$ & 2340 & 5787 & -3602 & 113 \\
\hline $\mathrm{H}(33 \mathrm{C})$ & 2432 & 7076 & -3161 & 113 \\
\hline $\mathrm{H}(35 \mathrm{~A})$ & 810 & 1544 & -1824 & 52 \\
\hline $\mathrm{H}(35 \mathrm{~B})$ & 1991 & 2105 & -805 & 52 \\
\hline $\mathrm{H}(36 \mathrm{~A})$ & 2368 & 1126 & -2128 & 68 \\
\hline
\end{tabular}




\begin{tabular}{lrrrr}
$\mathrm{H}(36 \mathrm{~B})$ & 3347 & 2540 & -1671 & 68 \\
$\mathrm{H}(36 \mathrm{C})$ & 2170 & 1980 & -2686 & 68 \\
$\mathrm{H}(38 \mathrm{~A})$ & 1947 & 4958 & 3167 & 47 \\
$\mathrm{H}(39 \mathrm{~A})$ & 2654 & 4226 & 4323 & 59 \\
$\mathrm{H}(40 \mathrm{~A})$ & 4397 & 5533 & 5770 & 67 \\
$\mathrm{H}(41 \mathrm{~A})$ & 5423 & 7590 & 6088 & 69 \\
$\mathrm{H}(42 \mathrm{~A})$ & 4734 & 8343 & 4932 & 52 \\
$\mathrm{H}(44 \mathrm{~A})$ & 2915 & 11864 & 5182 & 46 \\
$\mathrm{H}(45 \mathrm{~A})$ & 2195 & 12976 & 6098 & 61 \\
$\mathrm{H}(46 \mathrm{~A})$ & 325 & 12927 & 5329 & 60 \\
$\mathrm{H}(47 \mathrm{~A})$ & -817 & 11816 & 3645 & 57 \\
$\mathrm{H}(48 \mathrm{~A})$ & -98 & 10743 & 2721 & 46 \\
\hline
\end{tabular}




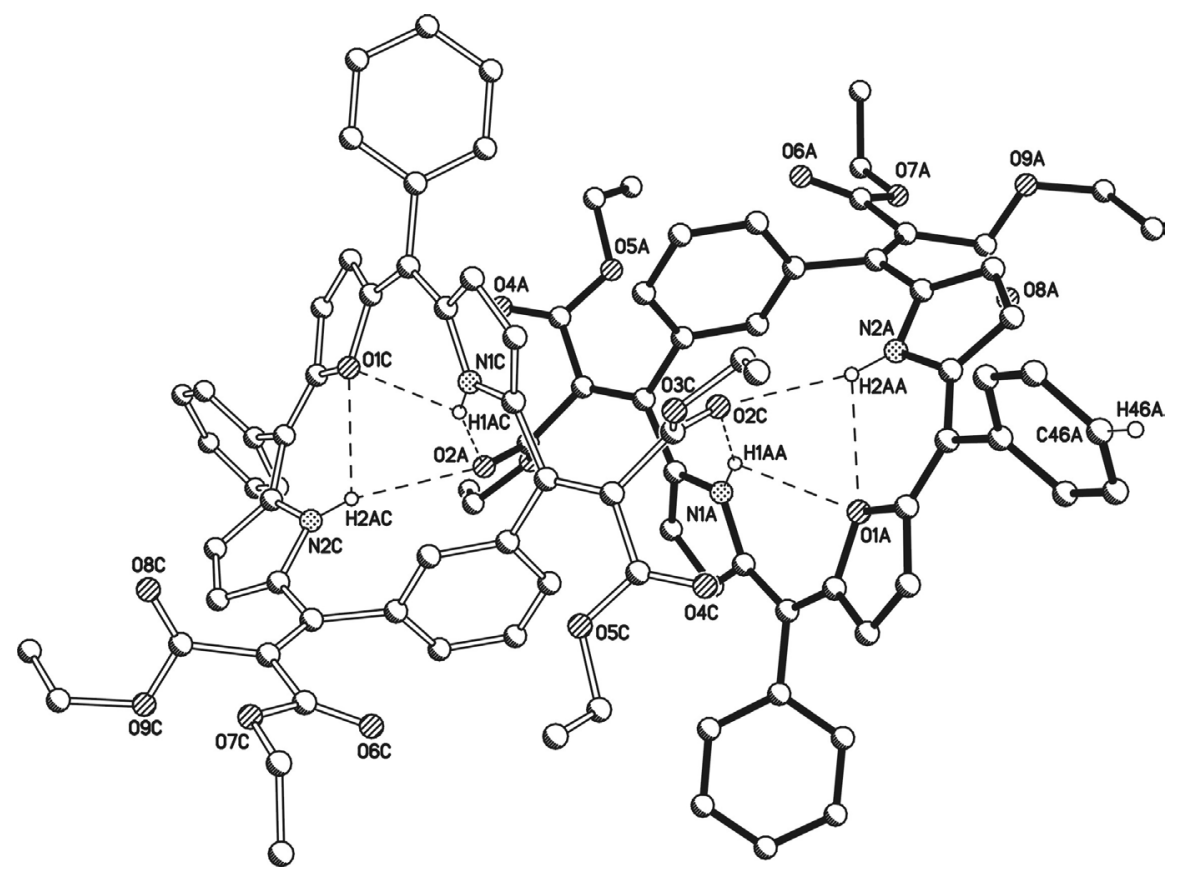

Figure S1. Intra- and intermolecular H-bonds for 10. (A: original atoms, C: $-x,-y+1,-z$ )

$\begin{array}{lccccl}\text { D-H } & \mathrm{d}(\mathrm{D}-\mathrm{H}) & \mathrm{d}(\mathrm{H} . . \mathrm{A}) & <\mathrm{DHA} & \mathrm{d}(\mathrm{D} . . \mathrm{A}) & \mathrm{A} \\ \text { N1-H1A } & 0.88 & 2.140 & 153.84 & 2.955(3) & \mathrm{O} 2[-\mathrm{x},-\mathrm{y}+1,-\mathrm{z}] \\ \text { N1-H1A } & 0.88 & 2.610 & 113.32 & 3.067(3) & \mathrm{O} 1 \\ \text { N2-H2A } & 0.88 & 2.438 & 111.90 & 2.884(3) & \mathrm{O} 1 \\ \text { N2-H2A } & 0.88 & 2.633 & 141.43 & 3.366(3) & \text { O2 [-x, -y+1, -z }] \\ \text { C46-H46A } & 0.95 & 2.478 & 144.50 & 3.298(4) & \mathrm{O} 4[\mathrm{x}, \mathrm{y}+1, \mathrm{z}+1]\end{array}$




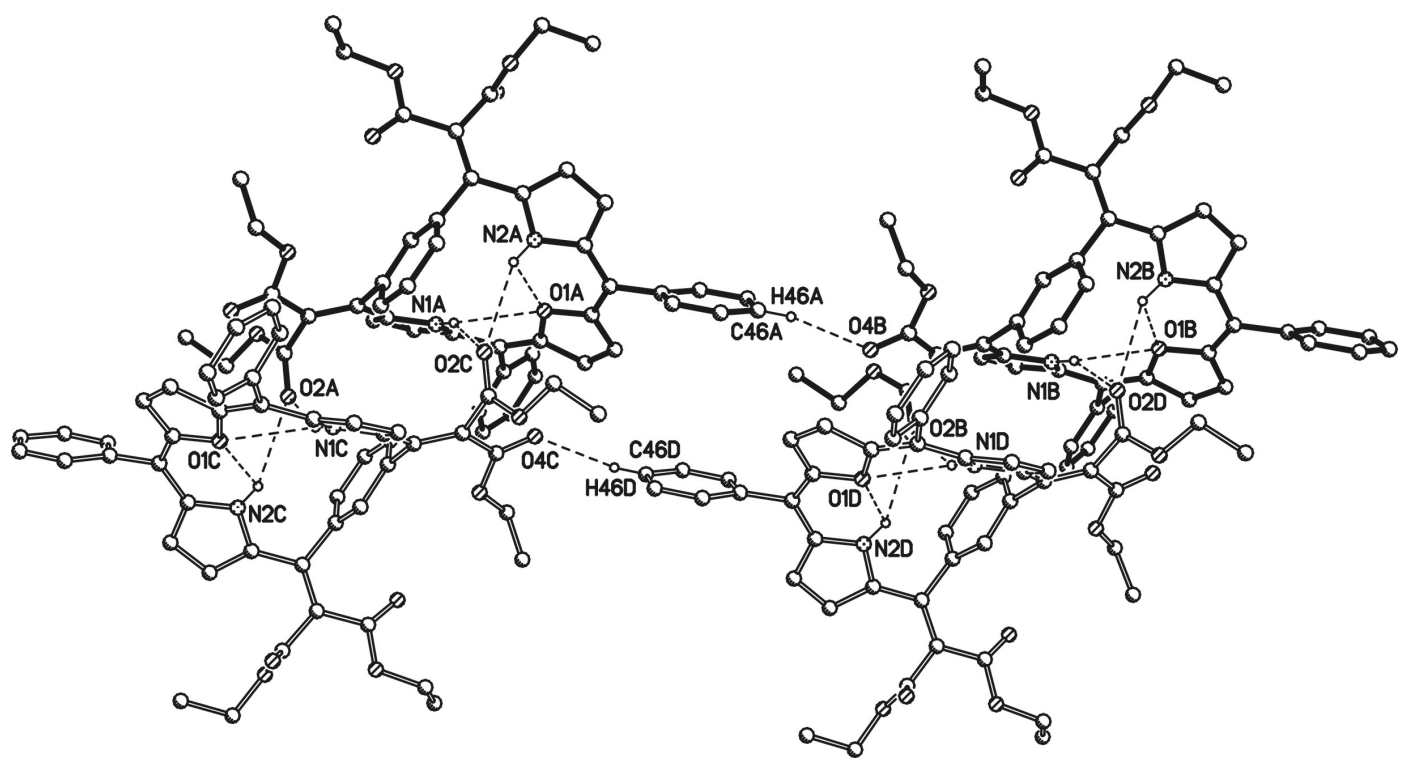

Figure S2. Intermolecular C-H...O hydrogen bonds for 10. A, orginal atom; B, $x, y+1, z+1 ; \mathrm{C},-x,-y+1,-z$; D: $-x,-y+2,-z+1$

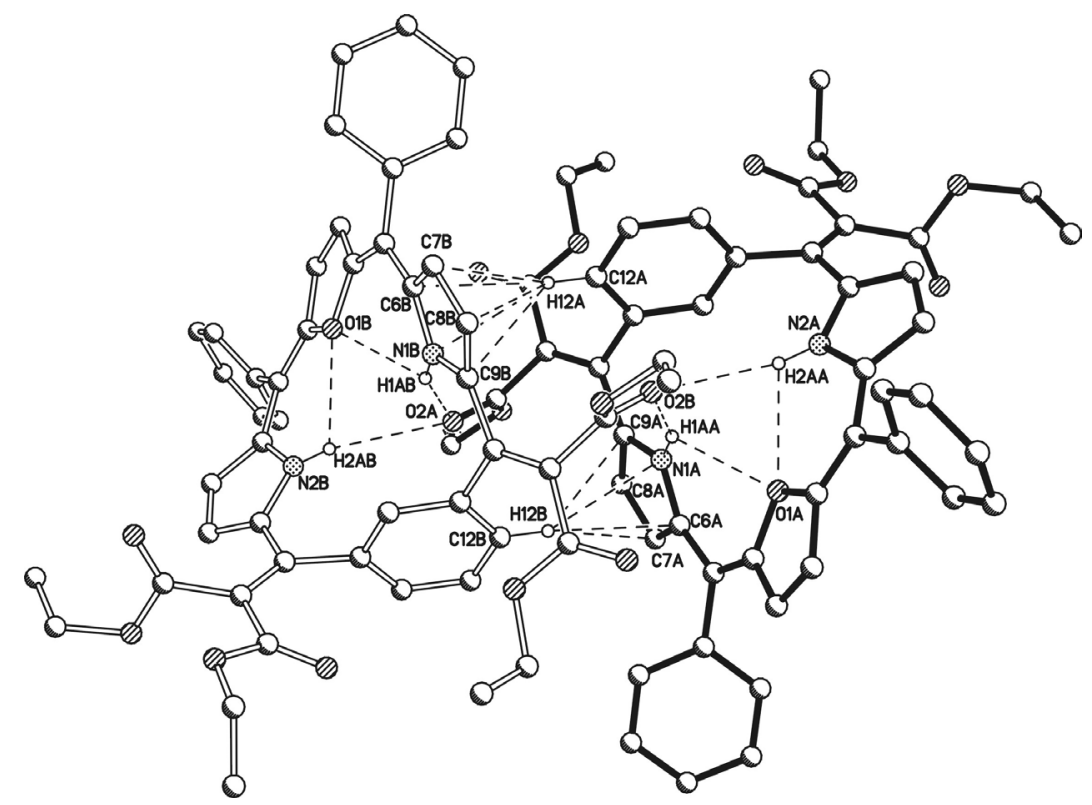

Figure S3. Intra- and intermolecular interactions for 10. $(\mathrm{C}(\pi) \cdots \mathrm{H}, \mathrm{N} \cdots \mathrm{H}$ and $\mathrm{O} \cdots \mathrm{H}$ interactions)

$\begin{array}{llllll}\text { C12A - N1B } & 3.532(4) & \text { C12A - C6B } & 3.597(4) & \text { C12A - C7B } & 3.604(4) \\ \text { C12A - C8B } & 3.533(4) & \text { C12A - C9B } & 3.468(4) & & \\ & & & & & \\ \text { H12A - N1B } & 2.6478 & \text { H12A - C6B } & 2.6585 & \text { H12A-C7B } & 2.8185 \\ \text { H12A - C8B } & 2.8966 & \text { H12A - C9B } & 2.7764 & & \end{array}$

The dihedral angle between phenyl ring $(\mathrm{C} 12 \mathrm{~A})$ and pyrrole ring(N1C, $\mathrm{C} 6 \mathrm{C}, \sim \mathrm{C} 9 \mathrm{C}): 66.9(1)^{\circ}$. 


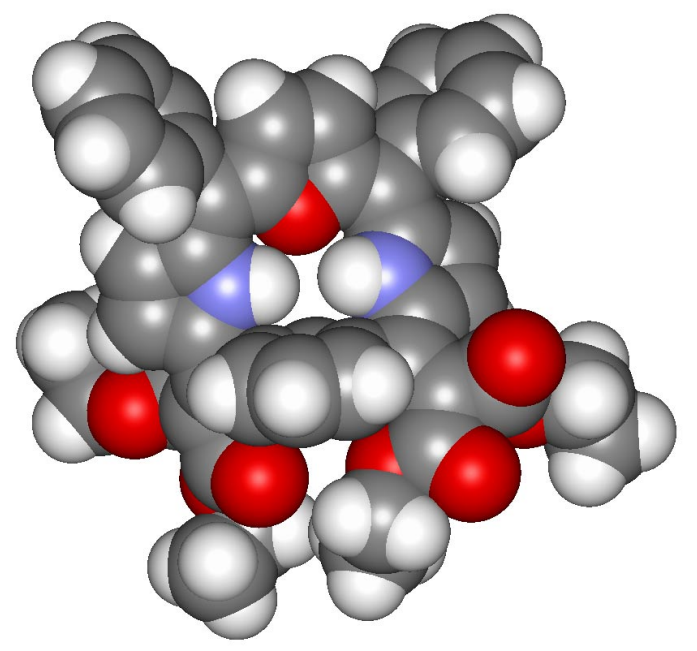

Figure S4. Space-filling model of $\mathbf{1 0 .}$
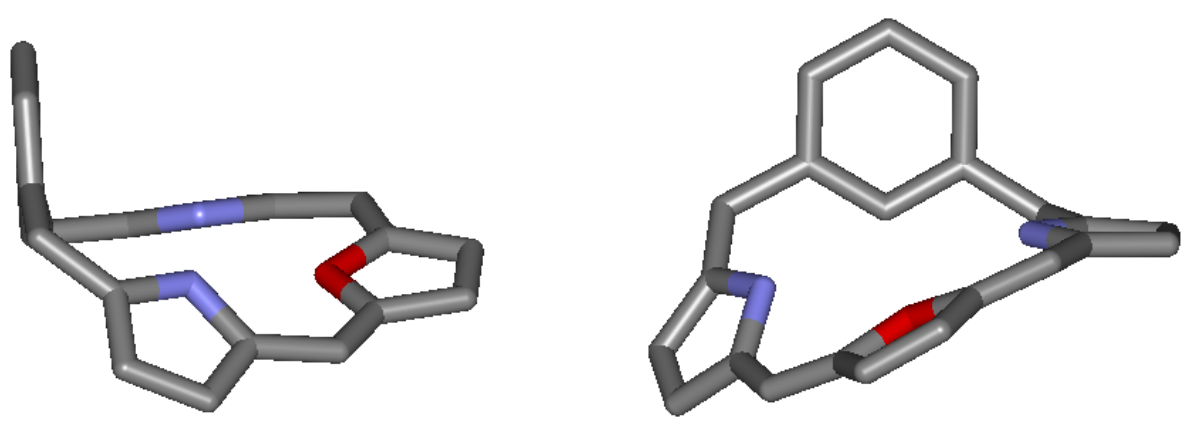

Figure S5. Perspective view of core unit for $\mathbf{1 0 .}$ 
Table 1. Crystal data and structure refinement for $\mathbf{1 1}$.

Empirical formula

Formula weight

Temperature

Wavelength

Crystal system

Space group

Unit cell dimensions

Volume

$\mathrm{Z}$

Density (calculated)

Absorption coefficient

$\mathrm{F}(000)$

Crystal size

Theta range for data collection

Index ranges

Reflections collected

Independent reflections

Completeness to theta $=28.30^{\circ}$

Refinement method

Data / restraints / parameters

Goodness-of-fit on $\mathrm{F}^{2}$

Final $\mathrm{R}$ indices $[\mathrm{I}>2 \operatorname{sigma}(\mathrm{I})]$

$\mathrm{R}$ indices (all data)

Largest diff. peak and hole
C47 H41 N3 O9

791.83

173(2) K

$0.71073 \AA$

Triclinic

P-1

$\mathrm{a}=12.1293(7) \AA \quad \square=68.8260(10)^{\circ}$.

$\mathrm{b}=13.3588(8) \AA \quad \square=83.0250(10)^{\circ}$.

$\mathrm{c}=13.7337(8) \AA \quad \square=75.2970(10)^{\circ}$.

2006.0(2) $\AA^{3}$

2

$1.311 \mathrm{Mg} / \mathrm{m}^{3}$

$0.091 \mathrm{~mm}^{-1}$

832

$0.30 \times 0.20 \times 0.20 \mathrm{~mm}^{3}$

1.59 to $28.30^{\circ}$.

$-13<=\mathrm{h}<=16,-16<=\mathrm{k}<=17,-17<=\mathrm{l}<=15$

12882

$8987[\mathrm{R}($ int $)=0.0608]$

$90.1 \%$

Full-matrix least-squares on $\mathrm{F}^{2}$

8987 / 0 / 532

0.911

$\mathrm{R} 1=0.0610, \mathrm{wR} 2=0.1359$

$\mathrm{R} 1=0.1738, \mathrm{wR} 2=0.1794$

0.807 and -0.442 e. $\AA^{-3}$ 
Table 2. Atomic coordinates $\left(\mathrm{x} 10^{4}\right)$ and equivalent isotropic displacement parameters $\left(\AA^{2} \times 10^{3}\right)$ for 11. $U(e q)$ is defined as one third of the trace of the orthogonalized $U^{\mathrm{ij}}$ tensor.

\begin{tabular}{|c|c|c|c|c|}
\hline & $\mathrm{x}$ & $\mathrm{y}$ & $\mathrm{z}$ & $\mathrm{U}(\mathrm{eq})$ \\
\hline $\mathrm{O}(1)$ & $7712(2)$ & $6719(2)$ & 2312(2) & $28(1)$ \\
\hline $\mathrm{O}(2)$ & $3110(2)$ & $3978(2)$ & $5315(2)$ & $34(1)$ \\
\hline $\mathrm{O}(3)$ & $1636(2)$ & $5040(2)$ & $4299(2)$ & $43(1)$ \\
\hline $\mathrm{O}(4)$ & $1324(2)$ & $5956(2)$ & $6059(2)$ & $50(1)$ \\
\hline $\mathrm{O}(5)$ & $1918(2)$ & 7506(2) & $5207(2)$ & $54(1)$ \\
\hline $\mathrm{O}(6)$ & $2596(2)$ & $10311(2)$ & $3801(2)$ & $65(1)$ \\
\hline $\mathrm{O}(7)$ & $2395(2)$ & $11376(2)$ & $2124(2)$ & $49(1)$ \\
\hline $\mathrm{O}(8)$ & $4587(2)$ & $12353(2)$ & $1727(2)$ & $46(1)$ \\
\hline $\mathrm{O}(9)$ & $4743(2)$ & $11144(2)$ & $900(2)$ & $38(1)$ \\
\hline $\mathrm{N}(1)$ & $5486(2)$ & $5964(2)$ & $3165(2)$ & $25(1)$ \\
\hline $\mathrm{N}(2)$ & $4359(2)$ & $8024(2)$ & $3803(2)$ & $27(1)$ \\
\hline $\mathrm{N}(3)$ & $6925(2)$ & $8619(2)$ & $2873(2)$ & $28(1)$ \\
\hline $\mathrm{C}(1)$ & $5796(3)$ & $5623(2)$ & 2319(2) & $24(1)$ \\
\hline $\mathrm{C}(2)$ & $4833(3)$ & $5427(3)$ & 2042(2) & $28(1)$ \\
\hline $\mathrm{C}(3)$ & $3934(3)$ & $5637(3)$ & 2733(2) & $30(1)$ \\
\hline $\mathrm{C}(4)$ & $4357(3)$ & 5971(3) & $3441(2)$ & $26(1)$ \\
\hline C(5) & $3745(3)$ & 6315(3) & $4276(2)$ & $25(1)$ \\
\hline $\mathrm{C}(6)$ & $4176(3)$ & 7107(3) & $4582(2)$ & $26(1)$ \\
\hline $\mathrm{C}(7)$ & 4403(3) & 6870(3) & $5613(2)$ & $28(1)$ \\
\hline $\mathrm{C}(8)$ & $4855(3)$ & 7583(3) & 5880(3) & $34(1)$ \\
\hline $\mathrm{C}(9)$ & $5063(3)$ & $8516(3)$ & 5092(3) & $35(1)$ \\
\hline$C(10)$ & 4772(3) & 8713(3) & 4078(3) & $29(1)$ \\
\hline$C(11)$ & $5033(3)$ & $9695(3)$ & $3200(3)$ & $32(1)$ \\
\hline$C(12)$ & $6250(3)$ & 9584(3) & 2914(3) & $31(1)$ \\
\hline$C(13)$ & 6924(3) & 10332(3) & 2646(3) & $45(1)$ \\
\hline$C(14)$ & $8021(3)$ & $9800(3)$ & 2446(3) & $41(1)$ \\
\hline$C(15)$ & $8006(3)$ & $8730(3)$ & 2563(3) & $30(1)$ \\
\hline$C(16)$ & 8910(3) & 7916(3) & 2306(3) & $31(1)$ \\
\hline$C(17)$ & 8771(3) & 7008(3) & 2165(3) & $29(1)$ \\
\hline$C(18)$ & $9546(3)$ & 6259(3) & 1733(3) & $35(1)$ \\
\hline$C(19)$ & $8976(3)$ & $5620(3)$ & $1545(3)$ & $31(1)$ \\
\hline
\end{tabular}




\begin{tabular}{|c|c|c|c|c|}
\hline$C(20)$ & $7812(3)$ & $5887(2)$ & $1896(2)$ & $26(1)$ \\
\hline $\mathrm{C}(21)$ & $6927(3)$ & $5472(2)$ & 1833(2) & $26(1)$ \\
\hline$C(22)$ & $2814(3)$ & $5929(3)$ & $4790(2)$ & $29(1)$ \\
\hline$C(23)$ & $2564(3)$ & $4879(3)$ & $4809(3)$ & $30(1)$ \\
\hline $\mathrm{C}(24)$ & $1216(3)$ & $4059(3)$ & $4457(3)$ & $56(1)$ \\
\hline $\mathrm{C}(25)$ & 1807(4) & $3447(4)$ & $3764(4)$ & $75(2)$ \\
\hline$C(26)$ & 1958(3) & $6444(3)$ & $5424(3)$ & $35(1)$ \\
\hline $\mathrm{C}(27)$ & 1067(4) & $8058(4)$ & $5803(5)$ & $86(2)$ \\
\hline $\mathrm{C}(28)$ & $1323(6)$ & $8937(6)$ & $5880(6)$ & $158(3)$ \\
\hline$C(29)$ & $4237(3)$ & $10561(3)$ & $2664(3)$ & $33(1)$ \\
\hline$C(30)$ & $3011(3)$ & $10720(3)$ & $2958(3)$ & $38(1)$ \\
\hline $\mathrm{C}(31)$ & 1177(3) & $11586(3)$ & $2255(3)$ & $54(1)$ \\
\hline$C(32)$ & $676(4)$ & $12338(5)$ & $1246(4)$ & $104(2)$ \\
\hline$C(33)$ & $4549(3)$ & $11469(3)$ & $1722(3)$ & $32(1)$ \\
\hline $\mathrm{C}(34)$ & $5054(3)$ & $11935(3)$ & $-91(3)$ & $40(1)$ \\
\hline$C(35)$ & $4808(3)$ & 11591(3) & $-947(3)$ & $48(1)$ \\
\hline$C(36)$ & $10045(3)$ & $8214(3)$ & 2048(3) & $37(1)$ \\
\hline$C(37)$ & $10723(4)$ & $8138(3)$ & $2819(4)$ & $69(2)$ \\
\hline $\mathrm{C}(38)$ & $11716(4)$ & $8502(4)$ & $2582(5)$ & $84(2)$ \\
\hline $\mathrm{C}(39)$ & $12069(4)$ & $8932(4)$ & $1557(5)$ & $71(2)$ \\
\hline $\mathrm{C}(40)$ & 11431(4) & $8989(4)$ & $780(4)$ & $75(2)$ \\
\hline $\mathrm{C}(41)$ & 10431(4) & $8633(4)$ & 1039(4) & $61(1)$ \\
\hline$C(42)$ & $7118(3)$ & 4801(3) & 1131(2) & $26(1)$ \\
\hline$C(43)$ & $7306(3)$ & $5314(3)$ & $66(3)$ & $37(1)$ \\
\hline $\mathrm{C}(44)$ & 7481(3) & $4724(3)$ & $-612(3)$ & $43(1)$ \\
\hline $\mathrm{C}(45)$ & $7479(3)$ & $3627(3)$ & $-232(3)$ & $40(1)$ \\
\hline$C(46)$ & $7285(3)$ & $3115(3)$ & $809(3)$ & $43(1)$ \\
\hline$C(47)$ & $7103(3)$ & $3696(3)$ & 1493(3) & $37(1)$ \\
\hline
\end{tabular}


Table 3. Bond lengths $[\AA]$ and angles $\left[{ }^{\circ}\right]$ for $\mathbf{1 1}$.

\begin{tabular}{|c|c|}
\hline $\mathrm{O}(1)-\mathrm{C}(20)$ & $1.395(3)$ \\
\hline $\mathrm{O}(1)-\mathrm{C}(17)$ & $1.405(4)$ \\
\hline $\mathrm{O}(2)-\mathrm{C}(23)$ & $1.214(4)$ \\
\hline $\mathrm{O}(3)-\mathrm{C}(23)$ & $1.329(4)$ \\
\hline $\mathrm{O}(3)-\mathrm{C}(24)$ & $1.460(4)$ \\
\hline $\mathrm{O}(4)-\mathrm{C}(26)$ & $1.207(4)$ \\
\hline $\mathrm{O}(5)-\mathrm{C}(26)$ & $1.329(4)$ \\
\hline $\mathrm{O}(5)-\mathrm{C}(27)$ & $1.457(5)$ \\
\hline $\mathrm{O}(6)-\mathrm{C}(30)$ & $1.196(4)$ \\
\hline $\mathrm{O}(7)-\mathrm{C}(30)$ & $1.337(4)$ \\
\hline $\mathrm{O}(7)-\mathrm{C}(31)$ & $1.434(4)$ \\
\hline $\mathrm{O}(8)-\mathrm{C}(33)$ & $1.197(4)$ \\
\hline $\mathrm{O}(9)-\mathrm{C}(33)$ & $1.325(4)$ \\
\hline $\mathrm{O}(9)-\mathrm{C}(34)$ & $1.466(4)$ \\
\hline $\mathrm{N}(1)-\mathrm{C}(1)$ & $1.373(4)$ \\
\hline $\mathrm{N}(1)-\mathrm{C}(4)$ & $1.374(4)$ \\
\hline $\mathrm{N}(2)-\mathrm{C}(10)$ & $1.331(4)$ \\
\hline $\mathrm{N}(2)-\mathrm{C}(6)$ & $1.353(4)$ \\
\hline $\mathrm{N}(3)-\mathrm{C}(15)$ & $1.356(4)$ \\
\hline $\mathrm{N}(3)-\mathrm{C}(12)$ & $1.357(4)$ \\
\hline$C(1)-C(2)$ & $1.385(4)$ \\
\hline $\mathrm{C}(1)-\mathrm{C}(21)$ & $1.451(4)$ \\
\hline$C(2)-C(3)$ & $1.398(4)$ \\
\hline$C(3)-C(4)$ & $1.399(4)$ \\
\hline$C(4)-C(5)$ & $1.442(4)$ \\
\hline $\mathrm{C}(5)-\mathrm{C}(22)$ & $1.371(4)$ \\
\hline$C(5)-C(6)$ & $1.491(4)$ \\
\hline$C(6)-C(7)$ & $1.380(4)$ \\
\hline $\mathrm{C}(7)-\mathrm{C}(8)$ & $1.383(4)$ \\
\hline $\mathrm{C}(8)-\mathrm{C}(9)$ & $1.381(4)$ \\
\hline $\mathrm{C}(9)-\mathrm{C}(10)$ & $1.393(4)$ \\
\hline$C(10)-C(11)$ & $1.501(4)$ \\
\hline $\mathrm{C}(11)-\mathrm{C}(29)$ & $1.342(4)$ \\
\hline $\mathrm{C}(11)-\mathrm{C}(12)$ & $1.464(5)$ \\
\hline
\end{tabular}




\begin{tabular}{|c|c|}
\hline$C(12)-C(13)$ & $1.371(4)$ \\
\hline$C(13)-C(14)$ & $1.386(5)$ \\
\hline$C(14)-C(15)$ & $1.384(4)$ \\
\hline$C(15)-C(16)$ & $1.446(5)$ \\
\hline$C(16)-C(17)$ & $1.349(4)$ \\
\hline$C(16)-C(36)$ & $1.495(5)$ \\
\hline$C(17)-C(18)$ & $1.432(4)$ \\
\hline $\mathrm{C}(18)-\mathrm{C}(19)$ & $1.332(4)$ \\
\hline $\mathrm{C}(19)-\mathrm{C}(20)$ & $1.434(4)$ \\
\hline$C(20)-C(21)$ & $1.352(4)$ \\
\hline$C(21)-C(42)$ & $1.499(4)$ \\
\hline$C(22)-C(26)$ & $1.470(5)$ \\
\hline$C(22)-C(23)$ & $1.498(4)$ \\
\hline$C(24)-C(25)$ & $1.478(6)$ \\
\hline$C(27)-C(28)$ & $1.331(7)$ \\
\hline $\mathrm{C}(29)-\mathrm{C}(30)$ & $1.474(5)$ \\
\hline C(29)-C(33) & $1.506(5)$ \\
\hline$C(31)-C(32)$ & $1.479(6)$ \\
\hline $\mathrm{C}(34)-\mathrm{C}(35)$ & $1.491(5)$ \\
\hline$C(36)-C(41)$ & $1.368(5)$ \\
\hline$C(36)-C(37)$ & $1.379(5)$ \\
\hline $\mathrm{C}(37)-\mathrm{C}(38)$ & $1.372(6)$ \\
\hline $\mathrm{C}(38)-\mathrm{C}(39)$ & $1.374(7)$ \\
\hline C(39)-C(40) & $1.361(7)$ \\
\hline $\mathrm{C}(40)-\mathrm{C}(41)$ & $1.375(5)$ \\
\hline $\mathrm{C}(42)-\mathrm{C}(47)$ & $1.382(4)$ \\
\hline $\mathrm{C}(42)-\mathrm{C}(43)$ & $1.391(4)$ \\
\hline $\mathrm{C}(43)-\mathrm{C}(44)$ & $1.389(5)$ \\
\hline $\mathrm{C}(44)-\mathrm{C}(45)$ & $1.367(5)$ \\
\hline $\mathrm{C}(45)-\mathrm{C}(46)$ & $1.365(5)$ \\
\hline $\mathrm{C}(46)-\mathrm{C}(47)$ & $1.387(5)$ \\
\hline $\mathrm{C}(20)-\mathrm{O}(1)-\mathrm{C}(17)$ & $107.7(2)$ \\
\hline $\mathrm{C}(23)-\mathrm{O}(3)-\mathrm{C}(24)$ & $116.0(3)$ \\
\hline $\mathrm{C}(26)-\mathrm{O}(5)-\mathrm{C}(27)$ & $115.8(3)$ \\
\hline $\mathrm{C}(30)-\mathrm{O}(7)-\mathrm{C}(31)$ & $117.7(3)$ \\
\hline
\end{tabular}




\begin{tabular}{|c|c|}
\hline $\mathrm{C}(33)-\mathrm{O}(9)-\mathrm{C}(34)$ & $117.0(3)$ \\
\hline $\mathrm{C}(1)-\mathrm{N}(1)-\mathrm{C}(4)$ & $110.4(2)$ \\
\hline $\mathrm{C}(10)-\mathrm{N}(2)-\mathrm{C}(6)$ & $116.3(3)$ \\
\hline $\mathrm{C}(15)-\mathrm{N}(3)-\mathrm{C}(12)$ & $110.8(3)$ \\
\hline $\mathrm{N}(1)-\mathrm{C}(1)-\mathrm{C}(2)$ & $106.8(3)$ \\
\hline $\mathrm{N}(1)-\mathrm{C}(1)-\mathrm{C}(21)$ & $126.3(3)$ \\
\hline $\mathrm{C}(2)-\mathrm{C}(1)-\mathrm{C}(21)$ & $126.9(3)$ \\
\hline $\mathrm{C}(1)-\mathrm{C}(2)-\mathrm{C}(3)$ & $108.5(3)$ \\
\hline $\mathrm{C}(2)-\mathrm{C}(3)-\mathrm{C}(4)$ & 107.4(3) \\
\hline $\mathrm{N}(1)-\mathrm{C}(4)-\mathrm{C}(3)$ & $106.8(3)$ \\
\hline $\mathrm{N}(1)-\mathrm{C}(4)-\mathrm{C}(5)$ & $125.0(3)$ \\
\hline$C(3)-C(4)-C(5)$ & $128.1(3)$ \\
\hline $\mathrm{C}(22)-\mathrm{C}(5)-\mathrm{C}(4)$ & $122.4(3)$ \\
\hline$C(22)-C(5)-C(6)$ & $120.4(3)$ \\
\hline $\mathrm{C}(4)-\mathrm{C}(5)-\mathrm{C}(6)$ & $117.2(3)$ \\
\hline $\mathrm{N}(2)-\mathrm{C}(6)-\mathrm{C}(7)$ & $123.2(3)$ \\
\hline $\mathrm{N}(2)-\mathrm{C}(6)-\mathrm{C}(5)$ & $116.7(3)$ \\
\hline$C(7)-C(6)-C(5)$ & 120.1(3) \\
\hline $\mathrm{C}(6)-\mathrm{C}(7)-\mathrm{C}(8)$ & 119.7(3) \\
\hline $\mathrm{C}(9)-\mathrm{C}(8)-\mathrm{C}(7)$ & $117.9(3)$ \\
\hline $\mathrm{C}(8)-\mathrm{C}(9)-\mathrm{C}(10)$ & $118.7(3)$ \\
\hline $\mathrm{N}(2)-\mathrm{C}(10)-\mathrm{C}(9)$ & 124.1(3) \\
\hline $\mathrm{N}(2)-\mathrm{C}(10)-\mathrm{C}(11)$ & $115.8(3)$ \\
\hline $\mathrm{C}(9)-\mathrm{C}(10)-\mathrm{C}(11)$ & $119.8(3)$ \\
\hline $\mathrm{C}(29)-\mathrm{C}(11)-\mathrm{C}(12)$ & $122.3(3)$ \\
\hline $\mathrm{C}(29)-\mathrm{C}(11)-\mathrm{C}(10)$ & $124.1(3)$ \\
\hline $\mathrm{C}(12)-\mathrm{C}(11)-\mathrm{C}(10)$ & $113.4(3)$ \\
\hline $\mathrm{N}(3)-\mathrm{C}(12)-\mathrm{C}(13)$ & 107.1(3) \\
\hline $\mathrm{N}(3)-\mathrm{C}(12)-\mathrm{C}(11)$ & $121.7(3)$ \\
\hline $\mathrm{C}(13)-\mathrm{C}(12)-\mathrm{C}(11)$ & $131.2(3)$ \\
\hline $\mathrm{C}(12)-\mathrm{C}(13)-\mathrm{C}(14)$ & $107.7(3)$ \\
\hline $\mathrm{C}(15)-\mathrm{C}(14)-\mathrm{C}(13)$ & $108.1(3)$ \\
\hline $\mathrm{N}(3)-\mathrm{C}(15)-\mathrm{C}(14)$ & $106.3(3)$ \\
\hline $\mathrm{N}(3)-\mathrm{C}(15)-\mathrm{C}(16)$ & $125.6(3)$ \\
\hline $\mathrm{C}(14)-\mathrm{C}(15)-\mathrm{C}(16)$ & $127.8(3)$ \\
\hline $\mathrm{C}(17)-\mathrm{C}(16)-\mathrm{C}(15)$ & $125.2(3)$ \\
\hline
\end{tabular}




\begin{tabular}{|c|c|}
\hline $\mathrm{C}(17)-\mathrm{C}(16)-\mathrm{C}(36)$ & $119.2(3)$ \\
\hline $\mathrm{C}(15)-\mathrm{C}(16)-\mathrm{C}(36)$ & $115.0(3)$ \\
\hline $\mathrm{C}(16)-\mathrm{C}(17)-\mathrm{O}(1)$ & $122.7(3)$ \\
\hline $\mathrm{C}(16)-\mathrm{C}(17)-\mathrm{C}(18)$ & 130.1(3) \\
\hline $\mathrm{O}(1)-\mathrm{C}(17)-\mathrm{C}(18)$ & $106.8(3)$ \\
\hline $\mathrm{C}(19)-\mathrm{C}(18)-\mathrm{C}(17)$ & 109.2(3) \\
\hline$C(18)-C(19)-C(20)$ & $108.6(3)$ \\
\hline $\mathrm{C}(21)-\mathrm{C}(20)-\mathrm{O}(1)$ & $123.6(3)$ \\
\hline $\mathrm{C}(21)-\mathrm{C}(20)-\mathrm{C}(19)$ & $129.0(3)$ \\
\hline $\mathrm{O}(1)-\mathrm{C}(20)-\mathrm{C}(19)$ & $107.4(3)$ \\
\hline $\mathrm{C}(20)-\mathrm{C}(21)-\mathrm{C}(1)$ & $128.7(3)$ \\
\hline $\mathrm{C}(20)-\mathrm{C}(21)-\mathrm{C}(42)$ & $116.0(3)$ \\
\hline $\mathrm{C}(1)-\mathrm{C}(21)-\mathrm{C}(42)$ & $115.2(3)$ \\
\hline $\mathrm{C}(5)-\mathrm{C}(22)-\mathrm{C}(26)$ & $125.7(3)$ \\
\hline $\mathrm{C}(5)-\mathrm{C}(22)-\mathrm{C}(23)$ & $123.5(3)$ \\
\hline $\mathrm{C}(26)-\mathrm{C}(22)-\mathrm{C}(23)$ & $110.8(3)$ \\
\hline $\mathrm{O}(2)-\mathrm{C}(23)-\mathrm{O}(3)$ & 124.1(3) \\
\hline $\mathrm{O}(2)-\mathrm{C}(23)-\mathrm{C}(22)$ & $122.4(3)$ \\
\hline $\mathrm{O}(3)-\mathrm{C}(23)-\mathrm{C}(22)$ & $113.2(3)$ \\
\hline $\mathrm{O}(3)-\mathrm{C}(24)-\mathrm{C}(25)$ & $112.6(3)$ \\
\hline $\mathrm{O}(4)-\mathrm{C}(26)-\mathrm{O}(5)$ & $123.1(4)$ \\
\hline $\mathrm{O}(4)-\mathrm{C}(26)-\mathrm{C}(22)$ & $123.0(4)$ \\
\hline $\mathrm{O}(5)-\mathrm{C}(26)-\mathrm{C}(22)$ & $113.8(3)$ \\
\hline $\mathrm{C}(28)-\mathrm{C}(27)-\mathrm{O}(5)$ & $113.6(5)$ \\
\hline $\mathrm{C}(11)-\mathrm{C}(29)-\mathrm{C}(30)$ & $123.5(3)$ \\
\hline $\mathrm{C}(11)-\mathrm{C}(29)-\mathrm{C}(33)$ & $121.5(3)$ \\
\hline $\mathrm{C}(30)-\mathrm{C}(29)-\mathrm{C}(33)$ & $114.9(3)$ \\
\hline $\mathrm{O}(6)-\mathrm{C}(30)-\mathrm{O}(7)$ & $123.3(4)$ \\
\hline $\mathrm{O}(6)-\mathrm{C}(30)-\mathrm{C}(29)$ & $126.7(3)$ \\
\hline $\mathrm{O}(7)-\mathrm{C}(30)-\mathrm{C}(29)$ & $110.0(3)$ \\
\hline $\mathrm{O}(7)-\mathrm{C}(31)-\mathrm{C}(32)$ & $108.4(3)$ \\
\hline $\mathrm{O}(8)-\mathrm{C}(33)-\mathrm{O}(9)$ & $126.0(3)$ \\
\hline $\mathrm{O}(8)-\mathrm{C}(33)-\mathrm{C}(29)$ & $124.2(3)$ \\
\hline $\mathrm{O}(9)-\mathrm{C}(33)-\mathrm{C}(29)$ & $109.8(3)$ \\
\hline $\mathrm{O}(9)-\mathrm{C}(34)-\mathrm{C}(35)$ & $107.3(3)$ \\
\hline $\mathrm{C}(41)-\mathrm{C}(36)-\mathrm{C}(37)$ & $116.7(4)$ \\
\hline
\end{tabular}




$\begin{array}{ll}\mathrm{C}(41)-\mathrm{C}(36)-\mathrm{C}(16) & 121.9(3) \\ \mathrm{C}(37)-\mathrm{C}(36)-\mathrm{C}(16) & 121.3(4) \\ \mathrm{C}(38)-\mathrm{C}(37)-\mathrm{C}(36) & 121.5(5) \\ \mathrm{C}(37)-\mathrm{C}(38)-\mathrm{C}(39) & 120.0(5) \\ \mathrm{C}(40)-\mathrm{C}(39)-\mathrm{C}(38) & 119.7(5) \\ \mathrm{C}(39)-\mathrm{C}(40)-\mathrm{C}(41) & 119.1(5) \\ \mathrm{C}(36)-\mathrm{C}(41)-\mathrm{C}(40) & 122.9(4) \\ \mathrm{C}(47)-\mathrm{C}(42)-\mathrm{C}(43) & 118.3(3) \\ \mathrm{C}(47)-\mathrm{C}(42)-\mathrm{C}(21) & 122.9(3) \\ \mathrm{C}(43)-\mathrm{C}(42)-\mathrm{C}(21) & 118.8(3) \\ \mathrm{C}(44)-\mathrm{C}(43)-\mathrm{C}(42) & 120.6(3) \\ \mathrm{C}(45)-\mathrm{C}(44)-\mathrm{C}(43) & 120.0(3) \\ \mathrm{C}(46)-\mathrm{C}(45)-\mathrm{C}(44) & 120.1(3) \\ \mathrm{C}(45)-\mathrm{C}(46)-\mathrm{C}(47) & 120.5(3) \\ \mathrm{C}(42)-\mathrm{C}(47)-\mathrm{C}(46) & 120.5(3)\end{array}$

Symmetry transformations used to generate equivalent atoms: 
Table 4. Anisotropic displacement parameters $\left(\AA^{2} \times 10^{3}\right)$ for 11. The anisotropic displacement factor exponent takes the form: $-2 \square^{2}\left[h^{2} a^{* 2} U^{11}+\ldots+2 h k a^{*} b^{*} U^{12}\right]$

\begin{tabular}{|c|c|c|c|c|c|c|}
\hline & $\mathrm{U}^{11}$ & $\mathrm{U}^{22}$ & $\mathrm{U}^{33}$ & $\mathrm{U}^{23}$ & $\mathrm{U}^{13}$ & $\mathrm{U}^{12}$ \\
\hline $\mathrm{O}(1)$ & $24(1)$ & $31(1)$ & $31(1)$ & $-14(1)$ & $0(1)$ & $-6(1)$ \\
\hline $\mathrm{O}(2)$ & $37(2)$ & $39(1)$ & $30(1)$ & $-14(1)$ & $-4(1)$ & $-12(1)$ \\
\hline $\mathrm{O}(3)$ & $35(2)$ & $56(2)$ & $44(2)$ & $-16(1)$ & $-11(1)$ & $-18(1)$ \\
\hline $\mathrm{O}(4)$ & $38(2)$ & $78(2)$ & $30(2)$ & $-15(1)$ & 11(1) & $-19(2)$ \\
\hline $\mathrm{O}(5)$ & $44(2)$ & $46(2)$ & $71(2)$ & $-28(2)$ & $16(1)$ & $-5(1)$ \\
\hline $\mathrm{O}(6)$ & $49(2)$ & $83(2)$ & $34(2)$ & $-3(2)$ & $8(1)$ & $7(2)$ \\
\hline $\mathrm{O}(7)$ & $30(2)$ & $69(2)$ & $34(2)$ & $-8(1)$ & $-2(1)$ & $-2(1)$ \\
\hline $\mathrm{O}(8)$ & $66(2)$ & $38(2)$ & $32(2)$ & $-10(1)$ & $-2(1)$ & $-14(1)$ \\
\hline $\mathrm{O}(9)$ & $54(2)$ & $31(1)$ & $24(1)$ & $-4(1)$ & $2(1)$ & $-11(1)$ \\
\hline $\mathrm{N}(1)$ & $26(2)$ & $30(2)$ & $22(2)$ & $-8(1)$ & $-2(1)$ & $-10(1)$ \\
\hline $\mathrm{N}(2)$ & $26(2)$ & $28(2)$ & $22(2)$ & $-6(1)$ & 1(1) & $-3(1)$ \\
\hline $\mathrm{N}(3)$ & $23(2)$ & $27(2)$ & $33(2)$ & $-11(1)$ & $1(1)$ & $-3(1)$ \\
\hline $\mathrm{C}(1)$ & $31(2)$ & $25(2)$ & $16(2)$ & $-7(1)$ & $-1(1)$ & $-6(2)$ \\
\hline $\mathrm{C}(2)$ & $35(2)$ & $36(2)$ & $17(2)$ & $-10(2)$ & $-2(1)$ & $-11(2)$ \\
\hline $\mathrm{C}(3)$ & $28(2)$ & $42(2)$ & $24(2)$ & $-12(2)$ & $-1(1)$ & $-13(2)$ \\
\hline $\mathrm{C}(4)$ & $23(2)$ & $35(2)$ & $21(2)$ & $-7(2)$ & $0(1)$ & $-10(2)$ \\
\hline $\mathrm{C}(5)$ & $25(2)$ & $31(2)$ & $16(2)$ & $-5(2)$ & $0(1)$ & $-5(2)$ \\
\hline $\mathrm{C}(6)$ & $26(2)$ & $29(2)$ & $20(2)$ & $-5(2)$ & 1(1) & $-6(2)$ \\
\hline $\mathrm{C}(7)$ & $36(2)$ & $27(2)$ & $20(2)$ & $-6(2)$ & $3(1)$ & $-9(2)$ \\
\hline $\mathrm{C}(8)$ & $46(2)$ & $30(2)$ & $24(2)$ & $-8(2)$ & $-3(2)$ & $-7(2)$ \\
\hline $\mathrm{C}(9)$ & $43(2)$ & $28(2)$ & $36(2)$ & $-12(2)$ & $-6(2)$ & $-7(2)$ \\
\hline$C(10)$ & $30(2)$ & $27(2)$ & $26(2)$ & $-7(2)$ & $1(2)$ & $-3(2)$ \\
\hline $\mathrm{C}(11)$ & $36(2)$ & $28(2)$ & $28(2)$ & $-9(2)$ & $4(2)$ & $-7(2)$ \\
\hline$C(12)$ & $34(2)$ & $24(2)$ & $30(2)$ & $-8(2)$ & $-1(2)$ & $-3(2)$ \\
\hline $\mathrm{C}(13)$ & $45(3)$ & $26(2)$ & $62(3)$ & $-17(2)$ & $9(2)$ & $-10(2)$ \\
\hline$C(14)$ & $33(2)$ & $32(2)$ & 61(3) & $-18(2)$ & $6(2)$ & $-12(2)$ \\
\hline$C(15)$ & $27(2)$ & $32(2)$ & $33(2)$ & $-13(2)$ & $-6(2)$ & $-4(2)$ \\
\hline$C(16)$ & $26(2)$ & $33(2)$ & $33(2)$ & $-9(2)$ & $-3(2)$ & $-7(2)$ \\
\hline$C(17)$ & $20(2)$ & $35(2)$ & $31(2)$ & $-11(2)$ & $-3(1)$ & $-5(2)$ \\
\hline$C(18)$ & $19(2)$ & $36(2)$ & $48(2)$ & $-18(2)$ & $1(2)$ & $-1(2)$ \\
\hline C(19) & $27(2)$ & $32(2)$ & $35(2)$ & $-14(2)$ & $3(2)$ & $-6(2)$ \\
\hline
\end{tabular}




\begin{tabular}{lllllll}
$\mathrm{C}(20)$ & $31(2)$ & $25(2)$ & $23(2)$ & $-11(2)$ & $0(1)$ & $-6(2)$ \\
$\mathrm{C}(21)$ & $28(2)$ & $24(2)$ & $24(2)$ & $-6(1)$ & $-2(1)$ & $-5(2)$ \\
$\mathrm{C}(22)$ & $24(2)$ & $35(2)$ & $25(2)$ & $-9(2)$ & $-1(1)$ & $-4(2)$ \\
$\mathrm{C}(23)$ & $29(2)$ & $43(2)$ & $21(2)$ & $-10(2)$ & $3(2)$ & $-14(2)$ \\
$\mathrm{C}(24)$ & $44(3)$ & $76(3)$ & $64(3)$ & $-32(3)$ & $-1(2)$ & $-32(2)$ \\
$\mathrm{C}(25)$ & $77(4)$ & $99(4)$ & $76(4)$ & $-47(3)$ & $-2(3)$ & $-41(3)$ \\
$\mathrm{C}(26)$ & $25(2)$ & $49(2)$ & $26(2)$ & $-8(2)$ & $-3(2)$ & $-8(2)$ \\
$\mathrm{C}(27)$ & $58(3)$ & $89(4)$ & $131(5)$ & $-81(4)$ & $16(3)$ & $2(3)$ \\
$\mathrm{C}(28)$ & $149(7)$ & $200(8)$ & $188(8)$ & $-143(7)$ & $86(6)$ & $-78(6)$ \\
$\mathrm{C}(29)$ & $30(2)$ & $36(2)$ & $25(2)$ & $-5(2)$ & $0(2)$ & $-4(2)$ \\
$\mathrm{C}(30)$ & $37(2)$ & $39(2)$ & $28(2)$ & $-7(2)$ & $0(2)$ & $-2(2)$ \\
$\mathrm{C}(31)$ & $29(2)$ & $71(3)$ & $54(3)$ & $-21(2)$ & $-1(2)$ & $-2(2)$ \\
$\mathrm{C}(32)$ & $44(3)$ & $152(5)$ & $80(4)$ & $-9(4)$ & $-21(3)$ & $2(3)$ \\
$\mathrm{C}(33)$ & $32(2)$ & $35(2)$ & $24(2)$ & $-9(2)$ & $-1(2)$ & $-1(2)$ \\
$\mathrm{C}(34)$ & $57(3)$ & $36(2)$ & $22(2)$ & $-2(2)$ & $7(2)$ & $-17(2)$ \\
$\mathrm{C}(35)$ & $58(3)$ & $45(2)$ & $30(2)$ & $-4(2)$ & $-6(2)$ & $-6(2)$ \\
$\mathrm{C}(36)$ & $27(2)$ & $29(2)$ & $54(3)$ & $-17(2)$ & $-3(2)$ & $-3(2)$ \\
$\mathrm{C}(37)$ & $55(3)$ & $65(3)$ & $76(3)$ & $14(2)$ & $-38(3)$ & $-32(2)$ \\
$\mathrm{C}(38)$ & $57(3)$ & $52(3)$ & $126(5)$ & $12(3)$ & $-54(3)$ & $-25(3)$ \\
$\mathrm{C}(39)$ & $33(3)$ & $47(3)$ & $140(5)$ & $-39(3)$ & $11(3)$ & $-15(2)$ \\
$\mathrm{C}(40)$ & $58(3)$ & $98(4)$ & $102(4)$ & $-67(3)$ & $38(3)$ & $-46(3)$ \\
$\mathrm{C}(41)$ & $54(3)$ & $88(3)$ & $71(3)$ & $-55(3)$ & $20(2)$ & $-37(3)$ \\
$\mathrm{C}(42)$ & $23(2)$ & $32(2)$ & $25(2)$ & $-12(2)$ & $0(1)$ & $-5(2)$ \\
$\mathrm{C}(43)$ & $43(2)$ & $38(2)$ & $33(2)$ & $-14(2)$ & $3(2)$ & $-12(2)$ \\
$\mathrm{C}(44)$ & $55(3)$ & $47(2)$ & $28(2)$ & $-16(2)$ & $5(2)$ & $-13(2)$ \\
$\mathrm{C}(45)$ & $41(2)$ & $46(2)$ & $41(2)$ & $-25(2)$ & $1(2)$ & $-11(2)$ \\
$\mathrm{C}(46)$ & $51(3)$ & $34(2)$ & $47(3)$ & $-17(2)$ & $2(2)$ & $-14(2)$ \\
$\mathrm{C}(47)$ & $46(2)$ & $31(2)$ & $31(2)$ & $-9(2)$ & $5(2)$ & $-12(2)$ \\
& & & & & & \\
\hline & & & & & & \\
\hline
\end{tabular}


Table 5. Hydrogen coordinates ( x 10 $0^{4}$ ) and isotropic displacement parameters $\left(\AA^{2} \mathrm{x} 10^{3}\right)$ for 11.

\begin{tabular}{|c|c|c|c|c|}
\hline & $\mathrm{x}$ & $\mathrm{y}$ & $\mathrm{z}$ & $\mathrm{U}(\mathrm{eq})$ \\
\hline $\mathrm{H}(1 \mathrm{~A})$ & 5945 & 6151 & 3484 & 31 \\
\hline $\mathrm{H}(3 \mathrm{~A})$ & 6693 & 8005 & 3027 & 33 \\
\hline $\mathrm{H}(2 \mathrm{~B})$ & 4789 & 5189 & 1477 & 34 \\
\hline $\mathrm{H}(3 \mathrm{~B})$ & 3176 & 5566 & 2725 & 36 \\
\hline $\mathrm{H}(7 \mathrm{~A})$ & 4249 & 6220 & 6135 & 34 \\
\hline $\mathrm{H}(8 \mathrm{~A})$ & 5017 & 7436 & 6585 & 40 \\
\hline $\mathrm{H}(9 \mathrm{~A})$ & 5398 & 9014 & 5239 & 42 \\
\hline $\mathrm{H}(13 \mathrm{~A})$ & 6684 & 11081 & 2604 & 54 \\
\hline $\mathrm{H}(14 \mathrm{~A})$ & 8669 & 10115 & 2261 & 50 \\
\hline $\mathrm{H}(18 \mathrm{~A})$ & 10339 & 6223 & 1602 & 41 \\
\hline $\mathrm{H}(19 \mathrm{~A})$ & 9289 & 5077 & 1230 & 37 \\
\hline $\mathrm{H}(24 \mathrm{~A})$ & 389 & 4280 & 4327 & 67 \\
\hline $\mathrm{H}(24 \mathrm{~B})$ & 1319 & 3566 & 5193 & 67 \\
\hline $\mathrm{H}(25 \mathrm{~A})$ & 1499 & 2796 & 3904 & 90 \\
\hline $\mathrm{H}(25 \mathrm{~B})$ & 2625 & 3215 & 3897 & 90 \\
\hline $\mathrm{H}(25 \mathrm{C})$ & 1689 & 3924 & 3034 & 90 \\
\hline $\mathrm{H}(27 \mathrm{~A})$ & 323 & 8289 & 5466 & 103 \\
\hline $\mathrm{H}(27 \mathrm{~B})$ & 983 & 7527 & 6514 & 103 \\
\hline $\mathrm{H}(28 \mathrm{~A})$ & 733 & 9254 & 6307 & 190 \\
\hline $\mathrm{H}(28 \mathrm{~B})$ & 1365 & 9487 & 5181 & 190 \\
\hline $\mathrm{H}(28 \mathrm{C})$ & 2062 & 8718 & 6208 & 190 \\
\hline $\mathrm{H}(31 \mathrm{~A})$ & 925 & 11927 & 2799 & 64 \\
\hline $\mathrm{H}(31 \mathrm{~B})$ & 922 & 10886 & 2477 & 64 \\
\hline $\mathrm{H}(32 \mathrm{~A})$ & -158 & 12492 & 1323 & 124 \\
\hline $\mathrm{H}(32 \mathrm{~B})$ & 924 & 11991 & 714 & 124 \\
\hline $\mathrm{H}(32 \mathrm{C})$ & 930 & 13029 & 1033 & 124 \\
\hline $\mathrm{H}(34 \mathrm{~A})$ & 5873 & 11933 & -113 & 48 \\
\hline $\mathrm{H}(34 \mathrm{~B})$ & 4602 & 12688 & -170 & 48 \\
\hline $\mathrm{H}(35 \mathrm{~A})$ & 5006 & 12105 & -1623 & 57 \\
\hline $\mathrm{H}(35 \mathrm{~B})$ & 3995 & 11597 & -918 & 57 \\
\hline
\end{tabular}




$\begin{array}{lrrrr}\mathrm{H}(35 \mathrm{C}) & 5261 & 10846 & -861 & 57 \\ \mathrm{H}(37 \mathrm{~A}) & 10497 & 7828 & 3531 & 83 \\ \mathrm{H}(38 \mathrm{~A}) & 12161 & 8457 & 3126 & 100 \\ \mathrm{H}(39 \mathrm{~A}) & 12755 & 9187 & 1391 & 85 \\ \mathrm{H}(40 \mathrm{~A}) & 11674 & 9272 & 70 & 90 \\ \mathrm{H}(41 \mathrm{~A}) & 9989 & 8681 & 491 & 74 \\ \mathrm{H}(43 \mathrm{~A}) & 7313 & 6075 & -200 & 45 \\ \mathrm{H}(44 \mathrm{~A}) & 7603 & 5083 & -1339 & 51 \\ \mathrm{H}(45 \mathrm{~A}) & 7614 & 3221 & -694 & 48 \\ \mathrm{H}(46 \mathrm{~A}) & 7273 & 2355 & 1067 & 51 \\ \mathrm{H}(47 \mathrm{~A}) & 6968 & 3331 & 2216 & 44 \\ \end{array}$




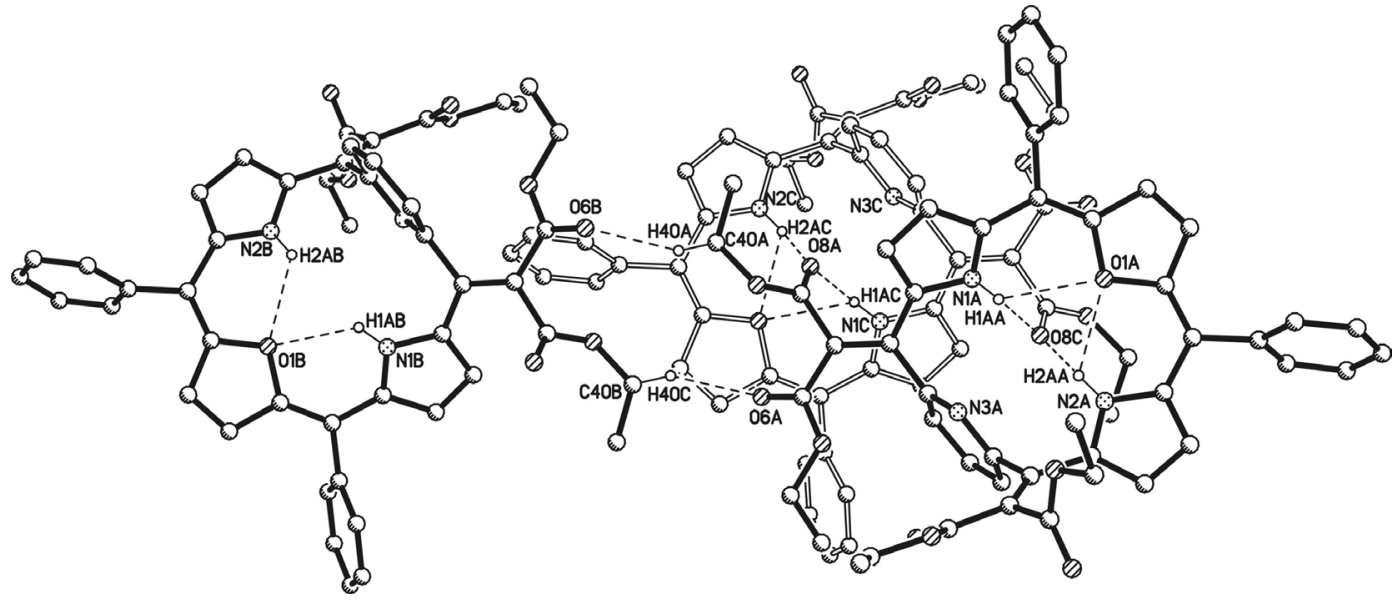

Figure S6. Intra- Intermolecular interactions between two adjacent molecules for 11. (A: original atom; B: $-x,-y+1,-z+1 ; \mathrm{C},-x+1,-y+1,-z+1)$.

$\begin{array}{lccccl}\text { D-H } & \mathrm{d}(\mathrm{D}-\mathrm{H}) & \mathrm{d}(\mathrm{H} . . \mathrm{A}) & <\mathrm{DHA} & \mathrm{d}(\mathrm{D} . . \mathrm{A}) & \mathrm{A} \\ \text { N1-H1A } & 0.88 & 2.053 & 156.87 & 2.883(3) & \mathrm{O} 8[-\mathrm{x}+1,-\mathrm{y}+1,-\mathrm{z}+1] \\ \text { N1-H1A } & 0.88 & 2.621 & 112.97 & 3.073(3) & \mathrm{O} 1 \\ \text { N2-H2A } & 0.88 & 2.300 & 117.89 & 2.821(3) & \text { O1 } \\ \text { N2-H2A } & 0.88 & 2.777 & 236.67 & 3.470(4) & \text { O8 [-x+1, -y+1, -z+1 ] } \\ \text { C40-H40A } & 0.99 & 2.328 & 154.66 & 3.250(5) & \text { O6 [-x, -y+1, -z+1 }]\end{array}$




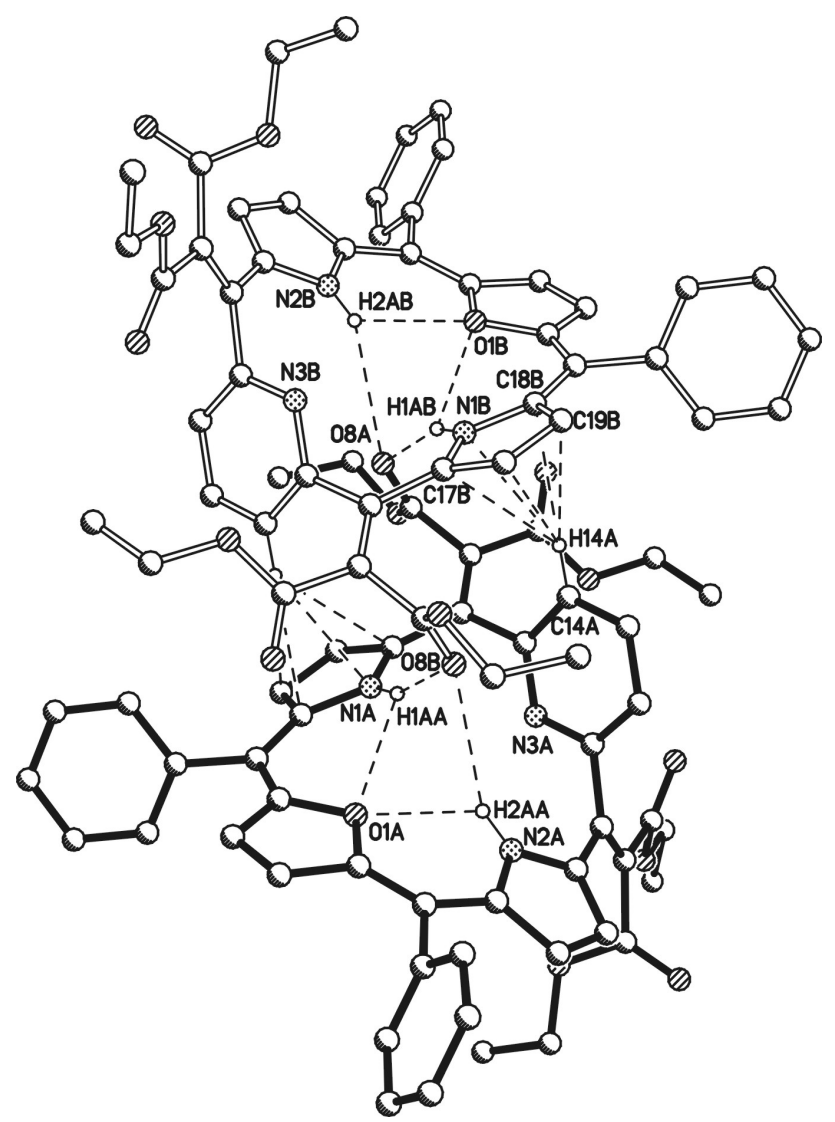

Figure S7. . Intra- and intermolecular interactions $(\mathrm{C}(\pi) \cdots \mathrm{H}, \mathrm{N} \cdots \mathrm{H}$ and $\mathrm{O} \cdots \mathrm{H}$ interactions $)$. A: original atoms; B: $-x+1,-y+1,-z+1$.

$\begin{array}{llll}\text { C14A-N1B } & 3.5169(0.0040) & \text { C14A-C17B } & 3.5171(0.0045) \\ \text { C14A-C18B } & 3.5529(0.0044) & \text { C14A-C19B } & 3.5762(0.0043) \\ \text { C14A-C20B } & 3.5540(0.0045) & & \end{array}$




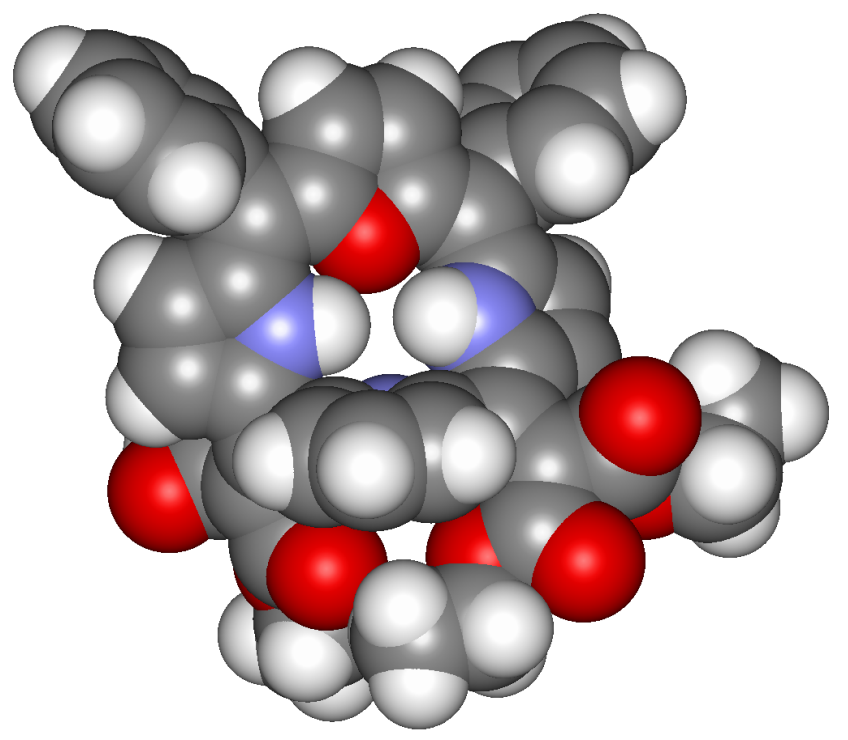

Figure S8. Space-filling model of $\mathbf{1 1 .}$
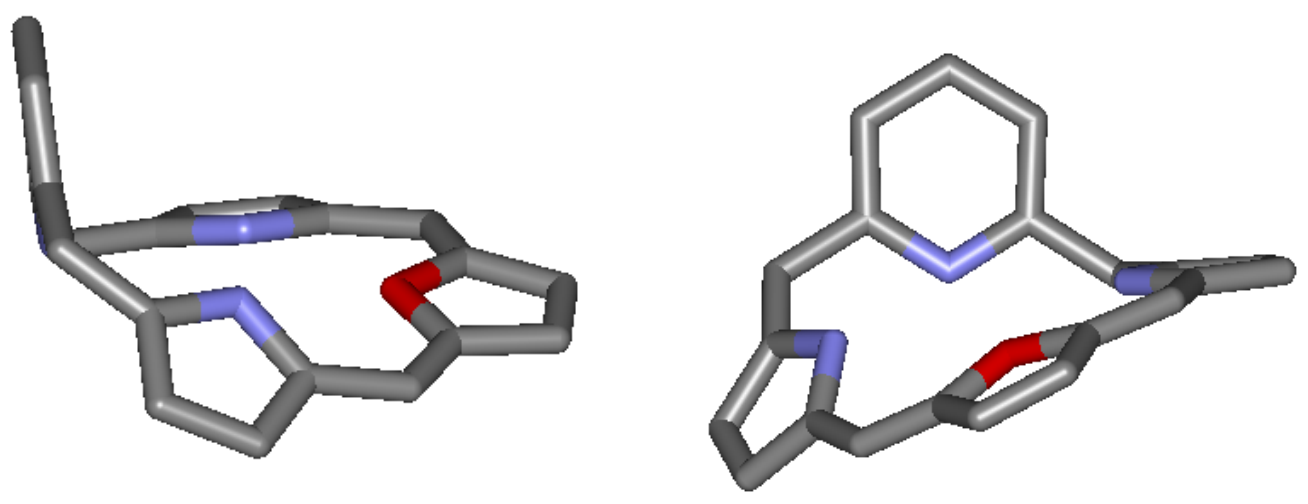

Figure S9. Perspective view of core unit for $\mathbf{1 1 .}$ 